\title{
AUCTIONS FOR OIL AND GAS LEASES WITH AN INFORMED BIDDER AND A RANDOM RESERVATION PRICE
}

\author{
By Kenneth Hendricks, Robert H. Porter, \\ AND Charles A. Wilson ${ }^{1}$
}

\begin{abstract}
We analyze a first-price, sealed bid auction with a random reservation price to study the federal sales of offshore oil and gas leases on drainage tracts. Our model assumes the object to be sold has an unknown common value, but one buyer has better information than the others. We permit the reservation price to be correlated with the information of the informed buyer, which reflects both his assessment of the value of the object and the probability of rejection at any bid. Assuming all random variables are affiliated, we establish the following results. (i) The percentage rate of increase in the distribution of the uninformed bid is never greater than the percentage rate of increase of the distribution of the informed bid. (ii) The distributions are identical at bids above the support of the reservation price. (iii) The informed buyer is more likely to submit low bids. We demonstrate that bid data from the federal sales of offshore drainage leases satisfy these restrictions.
\end{abstract}

KEYwords: Affiliation, auctions, bidding, oil.

\section{INTRODUCTION}

IN THIS PAPER WE ANALYZE a model of a first-price, sealed bid auction to study the federal sales of offshore oil and gas leases on drainage tracts. These tracts are adjacent to tracts on which deposits have already been discovered. Hendricks and Porter (1988) (HP henceforth) provide evidence that the value of these leases, while uncertain at the time of the sale, is approximately the same for all participants, but that the owner of an adjacent tract has superior information. Furthermore, Hendricks, Porter, and Spady (1989) (HPS henceforth) argue that, from the point of view of buyers, the reservation price of the seller is effectively a random variable, correlated with the bid of the informed bidder. We extend a model of Engelbrecht-Wiggans, Milgrom, and Weber (1983) (EMW henceforth) to incorporate these features and establish some restrictions on the equilibrium distributions of bids. We then test these implications with bid data for 295 drainage tracts off the coasts of Louisiana and Texas that were sold between 1959 and 1980 .

Previous studies of auctions with asymmetric information by Wilson (1967), Weverbergh (1979), and EMW were also motivated by the drainage auctions. The basic model is a sealed bid, first-price auction with a single informed buyer and one or more uninformed buyers bidding for an object of unknown but common value with a fixed reservation price. Under these assumptions, EMW demonstrate that the distributions of the high uninformed bid and the informed

\footnotetext{
${ }^{1}$ Support from the SSHRC, NSF Grant No. SES-8721231, and the C. V. Starr Center for Applied Economics is gratefully acknowledged. Expert research assistance has been provided by Robert Picard and Diana Whistler. We also thank Harry Paarsch, two conscientious referees, and the participants at several seminars for much valued input.
} 
bid are identical above the reservation price, with some informed bids possibly concentrated at the reservation price.

We extend the basic model to allow for an exogenous random reservation price that may be correlated with the value of the object and/or the information of the informed buyer. Consequently, to incorporate his estimate of the joint distribution of the value of the object, $V$, and the reservation price, $R$, conditional on his information, the signal of the informed buyer, $X$, must generally be multidimensional. This extension considerably complicates the analysis. To guarantee sufficient regularity conditions on the joint distribution of $(V, R, X)$, we require these random variables to be affiliated, a concept first introduced in the bidding literature by Milgrom and Weber (1982). This assumption implies that the conditional expected value of $V$ is nondecreasing in the realizations of both $R$ and $X$. It also implies that the distribution of $R$, conditional on $X$, satisfies the monotone likelihood ratio property with respect to the realizations of $X$.

With these restrictions, we establish that the equilibrium informed bid is a nondecreasing function of the signal $X$ and, consequently, also affiliated with $V$ and $R$. This relation in turn allows us to establish the following restrictions on the distribution functions of bids. First, the percentage rate of increase in the distribution function of the high uninformed bid is never greater than the percentage rate of increase in the distribution function of the informed bid. Second, above the support of $R$, the two distributions are identical. Third, near the lower bound of the support of $R$, only the informed buyer bids with positive probability. The high rejection rate for low bids forces the informed buyer to bid close to his valuation when it is low, which makes low bids by uninformed buyers unprofitable. Roughly speaking, these restrictions imply that uninformed buyers are less likely to participate than the informed buyer, but if they participate, they bid high rather than low.

Working with data on tracts sold before 1970, HP establish that neighbor firms, those that previously purchased leases on adjacent tracts, possessed considerable inside information about the value of the drainage tracts offered for sale. The average return to neighbor firms submitting a winning bid was roughly $180 \%$ of their bid, while the average return of nonneighbor firms was approximately equal to their bid. Also, after conditioning over relevant variables that were public information, the ex post discounted returns on drainage tracts sold before 1970 were highly correlated with the highest neighbor bid and essentially uncorrelated with the high nonneighbor bid. They also provide evidence of collusion among the neighbor firms, so that there was effectively only one informed bid. For example, the average high neighbor bid did not increase with the number of neighbor firms, nor was there a significant effect on nonneighbor bidding.

HPS analyze a superset of the HP data set that includes tracts sold between 1959 and 1979. On the basis of their analysis, they argue that the reservation price of the government had a significant exogenous random component. The 


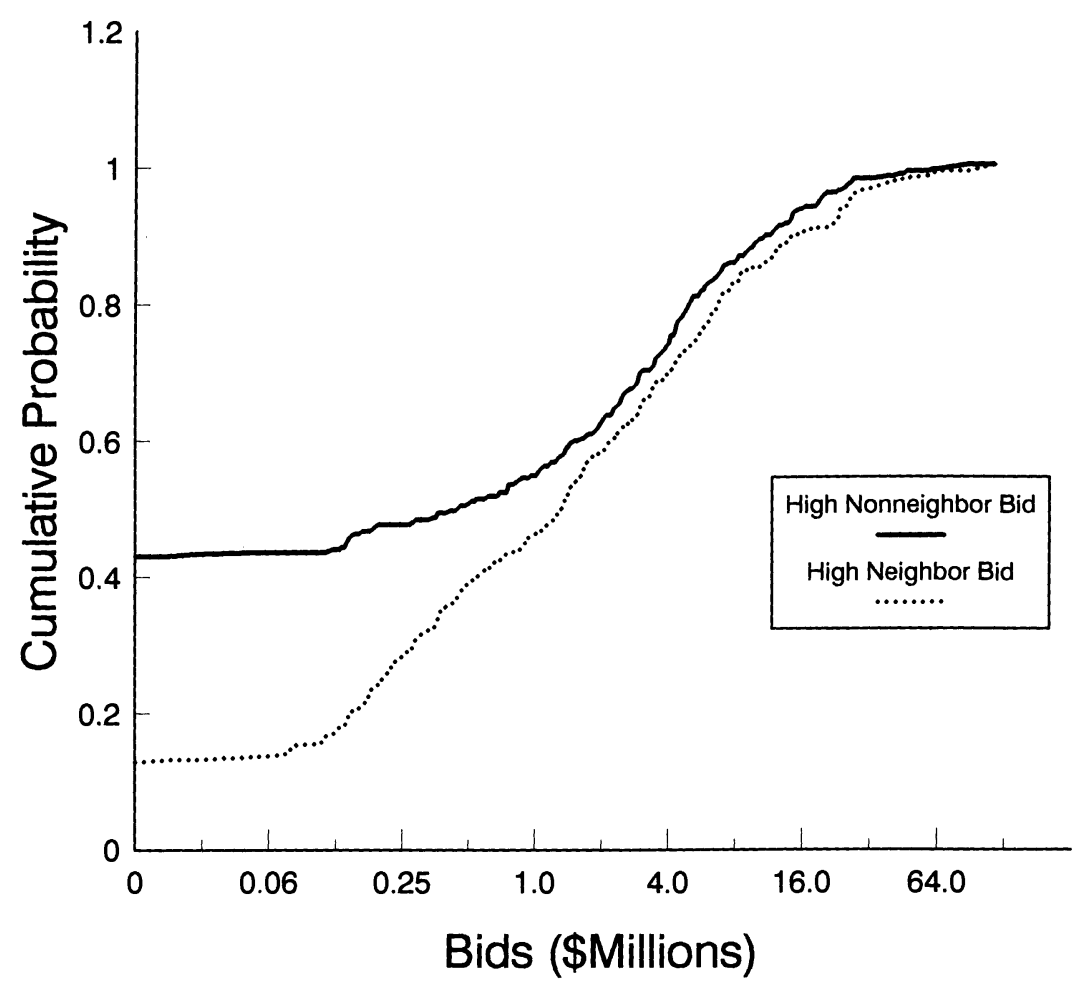

All bids are represented in 1972 dollars.

Figure 1.-Distribution of bids.

official minimum price was typically $\$ 25$ per acre. Nevertheless, the government rejected a higher bid on 58 of the 295 drainage tracts. Moreover, the rejection decision appears to be nonstrategic. Only about a third of the tracts in which the high bid was rejected were offered again at a later date, generally with a lag of 12 to 18 months. Also, the rejection option was rarely used in those instances when the government had the most to gain. Bids over five million dollars on drainage tracts were almost always accepted. HPS conclude that "the purpose of the government's rejection policy was to reduce the incentive that firms might have had to bid the preannounced minimum price on tracts that, on the basis of public information, were regarded as low value tracts."

Our working hypotheses in this paper are based on the conclusions drawn by HP and HPS from their analyses. We suppose that all neighbor firms collude as a single bidder and treat the highest neighbor bid as the informed bid. The nonneighbor firms are assumed to be uninformed bidders. Figure 1 illustrates the empirical distribution functions of the (logarithm of the) highest neighbor and nonneighbor bid on each tract in our sample. These distributions differ from the marginal distributions since our data include only those tracts on 
which at least one firm submitted a bid. For instance, the height of a neighbor distribution at 0 represents the proportion of tracts on which the neighbor firms submitted no bid among tracts which received at least one bid.

Notice that the three restrictions mentioned above appear to be satisfied. The percentage rate of increase in the distribution function of the high neighbor bid appears to be everywhere at least as great as the percentage rate of increase in the distribution function of the high nonneighbor bid. The distributions are roughly equal above 4 million dollars, where the high bid was rejected on only 6 of 122 tracts (as opposed to 58 rejections on the full sample of 295 tracts). Finally, neighbor firms were much more likely to submit relatively low bids than were nonneighbor firms. For example, more than $15 \%$ of the high neighbor bids lie between 0 and .25 million dollars, whereas less than $5 \%$ of the high nonneighbor bids lie in this range. We provide informal tests of these restrictions in Section 6.

The paper is organized as follows. In Section 2 we present the basic model and describe the auction. Section 3 contains an analysis of two examples to illustrate the main results of the paper. In Section 4, we consider the general model, and use the affiliation restriction to establish that the bid function of the informed buyer is monotonic in the signal $X$. Section 5 contains the main theoretical results of the paper described above. In Section 6, we investigate the extent to which the empirical distributions of high neighbor and high nonneighbor bids in the HPS data set satisfy our theoretical restrictions. Our conclusions are summarized in Section 7.

The proof that the bid function is monotonic in the information signal may be of some independent interest. It is apparently the first such result that allows for a multidimensional signal that may be correlated with both the value of the object and the seller's reservation price. However, as it stands, our argument does not extend to the case where there are two or more informed bidders. Since the reservation price is exogenous, its distribution does not satisfy various regularity conditions implied by profit maximization, nor does it vary with the bidding strategies of the buyers.

\section{THE UNDERLYING ENVIRONMENT AND THE AUCTION RULES}

An indivisible object with unknown value $V$ is to be sold in a sealed bid, first-price auction. The participants consist of an informed seller, who observes a private signal and sets a reservation price $R$ determined before the bids are revealed, an informed buyer, who observes a private signal $X$, and an uninformed buyer who observes only a public signal which we hold constant throughout. We restrict attention to a single uninformed buyer only to simplify notation. As we argue below, the equilibrium restrictions on the informed bidding strategy and the distribution of the highest uninformed bid are independent of the number of uninformed buyers. The buyers submit sealed bids without knowing the reservation price. A buyer wins the object with certainty if his bid is strictly greater than any other bid and not less than the reservation 
price. If both buyers make the same bid and it is not less than the reservation price, the object is allocated according to some rationing rule that is independent of the reservation price. The winner of the object receives the object in exchange for his bid.

We suppose that $V$ is a real valued random variable and $R$ is a positive random variable, both with finite expectation. The realization of $X$ lies in an $n$-dimensional Euclidian space. The joint distribution of $(V, R, X)$ is common knowledge. Throughout the paper, random variables and their associated probability distributions are denoted by upper case letters, real numbers by lower case letters, and functions by lower case Greek letters.

In equilibrium, the uninformed buyer must generally randomize his bid. Therefore, we suppose that he observes the realization of a random variable $U$ which is uniformly distributed on $[0,1]$ and independent of $(V, R, X)$. A strategy for the uninformed buyer is a real valued function $\alpha$ of the realizations of $U$ which, without loss of generality, we restrict to be nondecreasing. The probability induced by $\alpha(U)$ is denoted by $P_{\alpha}$ and the associated distribution function by $G_{\alpha}$. Similarly, a strategy for the informed buyer is a real valued function $\beta$ of the realizations of $X .^{2}$ The probability induced by $\beta(X)$ is denoted by $P_{\beta}$ and the associated distribution function by $G_{\beta}$.

\section{EQUILIBRIUM WITH AN INDEPENDENT RESERVATION PRICE: TWO EXAMPLES}

We begin with an informal discussion of the game when the reservation price is independent of both the true value and the signal of the informed buyer. We then analyze two simple examples to illustrate most of the properties of the equilibrium to be derived in a more general context in Section 5 .

When $R$ is independent of $V$ and $X$, and assuming $G_{\alpha}$ is continuous, the expected payoff to the informed buyer with signal $x$ who bids $b$ may be written as $E[V-b \mid X=x] K(b) G_{\alpha}(b)$, where $K(b)$ denotes the probability that the reservation price is not greater than $b$. In this case, the optimal bid of an informed buyer with signal $x$ depends only on the value of $H(X) \equiv E[V \mid X]$. The strategy of the informed buyer, $\beta$, is then simply a function of the realizations of $H$. This in turn simplifies the definition of payoffs for the uninformed buyer. With this simplification, the expected payoff to the uninformed buyer from bidding $b$ may be expressed as $E[V-b \mid \beta \leqslant b] K(b) G_{\beta}(b)$. An equilibrium is then a function $\beta$ and a distribution function $G_{\alpha}$ which are mutual best responses. In particular, the support of $G_{\alpha}$ must be contained in the set of bids which maximize $E[V-b \mid \beta \leqslant b] K(b) G_{\beta}(b)$.

In what follows, $\beta$ denotes the equilibrium bid function of the informed buyer. To determine $\beta$, it will be convenient to first solve for the optimal bid function for the informed buyer in the absence of an uninformed buyer. Let $\beta^{0}$ denote this function.

\footnotetext{
${ }^{2}$ If $X$ contains mass points, it may also be necessary for the informed bidder to randomize his bid. In this case, we may add a continuously distributed independent component to his signal. To simplify the notation, we avoid this complication. See Milgrom and Weber (1985) for further detail.
} 


\subsection{Example 1: $R$ is a Uniformly Distributed Random Variable}

Suppose $R$ is distributed uniformly on $[1,3]$ and $H$ is exponentially distributed with mean 6. (Note that, given the definition of $H$, the expected value of $H$ is also the (unconditional) expected value of the lease.) In the absence of an uninformed buyer, the problem of the informed buyer with valuation $h$ is to choose $b$ to maximize $(h-b) K(b)$. Solving for $b$ then yields the bidding strategy

$$
\beta^{0}(h)= \begin{cases}0 & h<1,3 \\ (h+1) / 2 & h \in[1,5], \\ 3 & h>5\end{cases}
$$

This bid function is illustrated in Figure 2a, with the value of $h$ represented on the vertical axis and the bid on the horizontal axis. If the conditional expected value of the object is less than 1 , the buyer does not bid. For values of $h$ between 1 and 5 , the bid function is increasing with slope $1 / 2$ and range $[1,3]$. For values of $h$ which exceed 5, the buyer bids 3, which is just sufficient to win the object with certainty.

Now consider the effect on the bid of an informed buyer of competition from an uninformed buyer. There are basically two steps to the analysis. The first step consists of showing that $\beta$ is nondecreasing which we demonstrate in Lemma 3 below. For the special case being analyzed here, a simple selfselection argument suffices (see EMW).

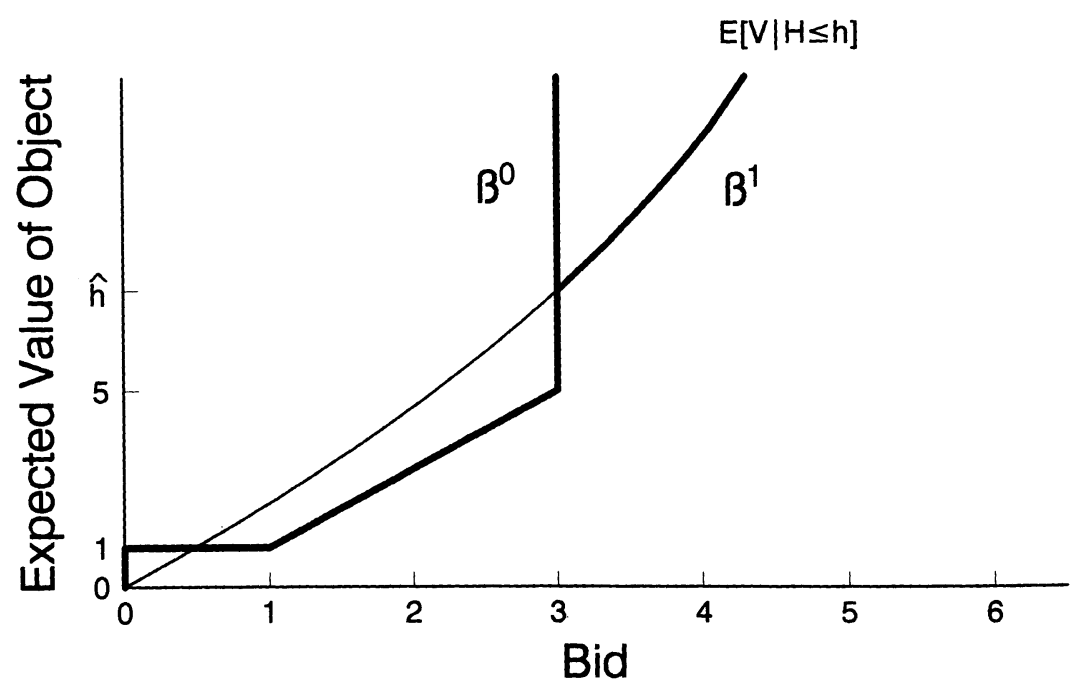

Figure 2a.-Bid functions for Example 1.

\footnotetext{
${ }^{3}$ Setting $\beta^{0}(h)=0$ for $h<1$ is just a convention. Any bid less than 1 will have the same effect-the buyer will never win the object.
} 
The second step is to show that the uninformed buyer cannot earn positive profits. This result is also standard although a careful argument is a bit tedious. The basic argument runs as follows. Let $b_{\alpha}$ be the lower bound of the support of $G_{\alpha}$ and suppose that $G_{\alpha}\left(b_{\alpha}\right)=0$. If the expected profit of the uninformed buyer is positive, then there must be a nondegenerate set of realizations of $H$ such that $h>b_{\alpha}$ and $\beta(h) \leqslant b_{\alpha}$. That is, for the uninformed buyer who bids slightly above $b_{\alpha}$, there must be some chance of winning when the expected value of the object exceeds $b_{\alpha}$. But, in that case, the informed buyer is not behaving optimally. Since he never wins the object with a bid of $b_{\alpha}$ or less, his optimal bid at realizations of $H$ larger than $b_{\alpha}$ must exceed $b_{\alpha}$. This contradiction establishes the result. A slight modification of the argument also can be applied in the case where $G_{\alpha}\left(b_{\alpha}\right)>0$.

The monotonicity of $\beta$ and the zero expected profit condition imply that, for $\beta(h)>0$,

$$
\beta(h) \geqslant E[V \mid H \leqslant h] .
$$

Since the uninformed buyer can always earn zero by not bidding, he never bids in the range where $\beta(h)$ exceeds $E[V \mid H \leqslant h]$. Consequently, $G_{\alpha}(\beta(h))=$ $G_{\alpha}(E[V \mid H \leqslant h])$. The definition of $\beta$ as a best response implies that $(h-$ $\beta(h)) K(\beta(h)) G_{\alpha}(\beta(h)) \geqslant(h-b) K(b) G_{\alpha}(b)$ for all $b$. Since $G_{\alpha}(b)$ is nondecreasing, it follows that $\beta(h)$ must also satisfy, for all $b \geqslant E[V \mid H \leqslant h]$,

$$
(h-\beta(h)) K(\beta(h)) \geqslant(h-b) K(b) .
$$

We conclude from relations (1) and (2) that $\beta(h)$ is the maximizer of $(h-b) K(b)$ subject to $b \geqslant E[V \mid H \leqslant h]$.

This characterization of $\beta$ reveals that the role of the uninformed buyer is essentially to impose a constraint on the bid function of the informed buyer. Conditional on realizations of the informed bid that are no greater than $b$, the conditional expected value of the object cannot exceed $b$. A single uninformed buyer is sufficient to impose this constraint, so there is no loss of generality in restricting attention to this case. Also, notice that, since $\beta^{0}(h)$ is the maximizer of $(h-b) K(b)$, it follows immediately that $\beta(h) \geqslant \beta^{0}(h)$. Both of these results do not depend upon the independence of $R$ and, in the Appendix, we show that they hold for any distribution of $(V, X, R)$.

Return now to Figure 2a. Here $\beta^{0}(h)$ exceeds $E[V \mid H \leqslant h]$ for values of $h$ between 1 and $\hat{h}$, where $\hat{h}$ is the unique solution to $E[V \mid H \leqslant \hat{h}]=3$. Therefore, over this interval, $\beta^{0}(h)$ also satisfies the conditions for $\beta(h)$. For larger values of $h, \beta^{0}(h)$ is equal to 3, which is less than $E[V \mid H \leqslant h]$. The uninformed buyer could then earn positive profit by submitting a bid between 3 and 6 , where the expected return is $6-b$. (A bid above 3 wins the object for sure against $\beta^{0}$, and 6 is the expected value of the lease.) Consequently, for valuations larger than $\hat{h}$, the constraint, $b \geqslant E[V \mid H \leqslant h]$, is binding and the informed buyer must increase his bid to $E[V \mid H \leqslant h]$. Faced with this revised strategy, the uninformed buyer is 


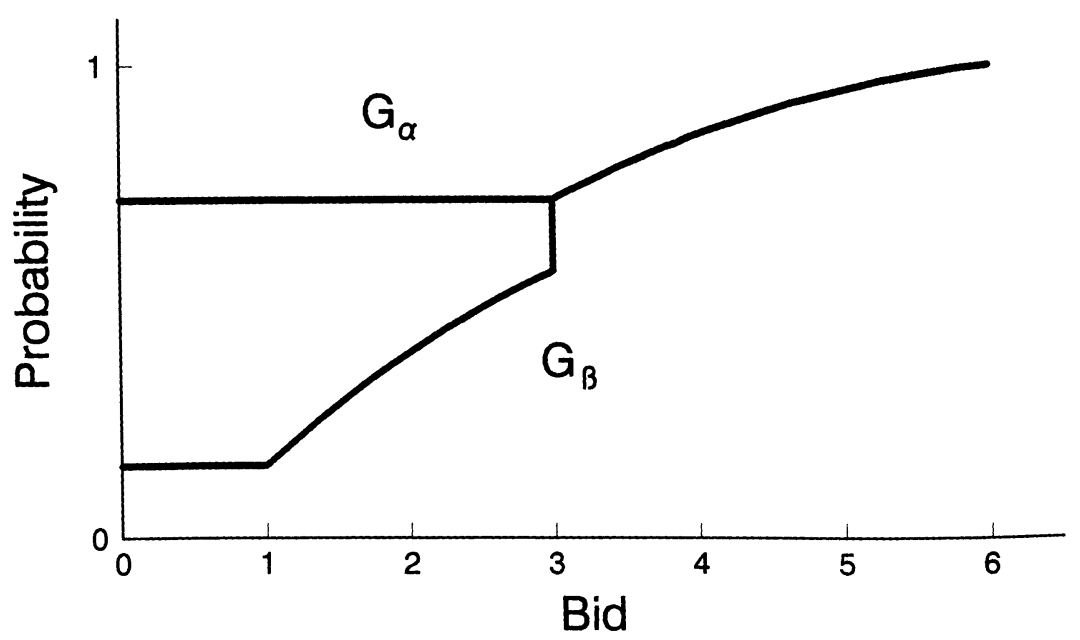

Figure 2b.-Distribution functions for Example 1.

now indifferent between submitting a bid between 3 and 6 and staying out. Consequently, his strategy can be adjusted so that the informed buyer has no incentive to deviate from $\beta$.

This example, although quite reasonable, is particularly well behaved. The support of the strategies of both buyers is a connected interval and $\beta(h)$ is equal to $E[V \mid H \leqslant h]$ at values of $h$ where the constraint, $b \geqslant E[V \mid H \leqslant h]$, is binding. The next example illustrates a case where neither of these conditions is satisfied.

\subsection{Example 2: $R$ is a Bernoulli Random Variable}

Suppose that $R$ has a Bernoulli distribution with equal mass concentrated at 1 and 3, and again assume that $H$ is exponentially distributed with mean 6 . In the absence of an uninformed buyer, the optimal bid function for the informed buyer is

$$
\beta^{0}(h)= \begin{cases}0, & h<1, \\ 1, & h \in[1,5], \\ 3, & h>5 .\end{cases}
$$

This bid function is illustrated in Figure 3a. If the expected value of the object is less than 1, the buyer does not bid. For values of $h$ between 1 and 5, expected profit is maximized by a bid of 1 which wins the object with probability $1 / 2$. For values of $h$ greater than 5 , the buyer bids 3 to win the object with certainty.

Now suppose an uninformed buyer enters the auction. Let $\tilde{h}$ be the unique solution to $E[V \mid H \leqslant \tilde{h}]=1$. For values of $h$ between $\tilde{h}$ and $5, \beta^{0}(h)$ is less than $E[V \mid H \leqslant h]$. Therefore, by bidding slightly above 1 , an uninformed buyer earns 


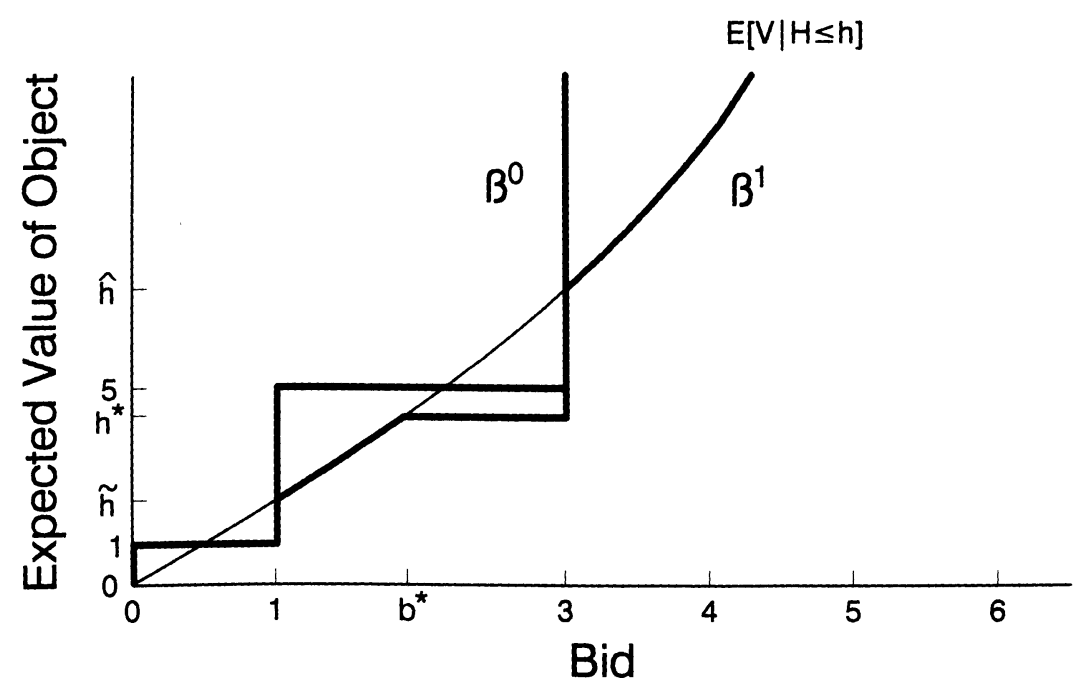

Figure 3a.-Bid functions for Example 2.

positive expected profits. Also, as in Example 1, a bid slightly above 3 yields positive profits as well. In this case, therefore, the constraint, $b \geqslant E[V \mid H \leqslant h]$, is binding on two intervals, $[\tilde{h}, 5]$ and $[\hat{h}, \infty)$. However, the necessary adjustment in the strategy of the uninformed buyer is not as straightforward as in Example 1. Relations (1) and (2) imply that when the realization of $h$ of $H$ lies between $\tilde{h}$ and 5, the informed buyer must choose between a bid of $E[V \mid H \leqslant h]$ and a bid of 3. This choice arises because the informed buyer's profit function is discontinuous at a bid of 3 , where the probability of beating the reservation price jumps from $1 / 2$ to 1 . Let $h^{*}$ denote the unique value of $h$ at which the informed buyer is indifferent between a bid of $E[V \mid H \leqslant h]$ and 3. It is easy to show that $h^{*}$ lies between $\tilde{h}$ and 5 . The equilibrium bid strategy is then defined as follows:

$$
\beta(h)= \begin{cases}\beta^{0}(h), & h<\tilde{h}, \\ E[V \mid H \leqslant h], & \tilde{h} \leqslant h \leqslant h^{*}, \\ 3, & h^{*}<h \leqslant \hat{h}, \\ E[V \mid H \leqslant h], & h>\hat{h} .\end{cases}
$$

\subsection{The Bid Distributions}

The distributions of the informed and uninformed bids for our two examples are illustrated in Figures $2 \mathrm{~b}$ and $3 \mathrm{~b}$. Although there is no closed form solution for the bid distributions, we may still establish a close relationship between the two distributions. ${ }^{4}$

\footnotetext{
${ }^{4}$ At points where $\beta$ is invertible, $G_{\beta}(b)=1-e^{-6 \beta^{-1}(b)}$. However, at points of increase in $\beta$, $\beta(h)=6-h /\left[e^{h / 6}-1\right]$, which does not yield a closed form inverse. Figures 2 and 3 were generated by using numerical approximations of $\beta^{-1}(b)$.
} 


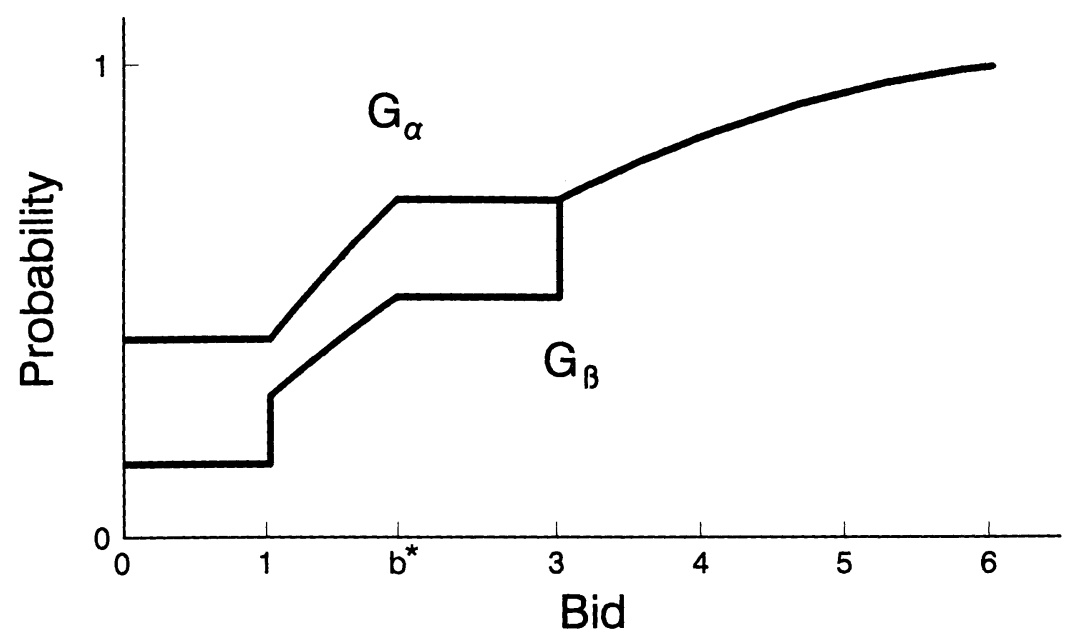

FIGURE 3b.-Distribution functions for Example 2.

Let $g_{\alpha}$ and $g_{\beta}$ denote the respective densities of $G_{\alpha}$ and $G_{\beta}$ and consider any bid $b$ in the interior of the support of both $G_{\alpha}$ and $G_{\beta}$. We note first that, in both examples, any such $b$ lies outside the support of $K$. Therefore, if the informed buyer raises his bid by one unit, his expected cost increases by $K G_{\alpha}$, the probability that he wins the object. Since $K$ is constant in some neighborhood of $b$, his only expected gain is from the additional chance of winning the object when the uninformed buyer bids just above $b,(h-b) \mathrm{Kg}_{\alpha}$. Consequently, the first order condition for informed profit maximization is $(h-b) K_{\alpha}=K G_{\alpha}$. Similarly, if the uninformed buyer increases his bid by one unit, his expected cost increases by $K G_{\beta}$ and his only gain is from winning the object when the informed buyer bids just above $b,(h-b) K_{\beta}$. Therefore, the first order condition for uninformed profit maximization is $(h-b) K g_{\beta}=K G_{\beta}$.

Comparison of the two first order conditions then yields $g_{\alpha} / G_{\alpha}=g_{\beta} / G_{\beta}$. Above the support of $R$, both distributions must have the same points of increase (otherwise, some buyer could lower his bid without loss). It follows immediately, therefore, that in both examples the distributions are identical for bids above 3. Furthermore, in Example 2, the rates of increase in the logs of the distribution functions are also equal on the interval [1, $\left.b^{*}\right]$.

In general, the presence of a random reservation price may result in an irregular distribution of bids. For instance, in Example 2, the supports of the distribution function of both buyers are not connected. Also, in both examples, there is at least one mass point at a positive bid in the distribution function of the informed bid. Notice, however, that in both cases the support of the informed bid contains the support of the uninformed bid. This property follows from the observation that the expected value of the object to a winning uninformed bid is the same at any price in an interval $\left(b^{\prime}, b\right)$ in which the informed buyer never bids. Therefore, if the expected profit to the uninformed buyer is zero at $b^{\prime}$, it must be negative at any higher bid in $\left(b^{\prime}, b\right)$. 
Also note that in both cases there is a bid $b$ such that $G_{\alpha}(b)=G_{\alpha}(0)$ and $G_{\beta}(b)>G_{\beta}(0)$. In Example $1, G_{\beta}$ is strictly increasing over [1,3], but $G_{\alpha}(3)=$ $G_{\alpha}(0)$. In Example 2, $G_{\alpha}$ is strictly increasing over [1, $\left.b^{*}\right]$, but $G_{\beta}$ has positive mass at 1 . This property is a consequence of the fact that at any positive bid submitted by the uninformed buyer, there must be some informed types that bid less, but whose valuations exceed the bid of the uninformed buyer. Otherwise, the uninformed buyer would earn negative profits, since he would win only when the value of the object is less than his bid.

In summary, in both examples the distribution of the uninformed bid stochastically dominates the distribution of the informed bid, and the distributions are equal above the support of the reservation price. In Example 1, there is a range of low bids in which only the informed buyer bids. In Example 2, the informed buyer bids the minimum reservation price with positive probability whereas the uninformed buyer always bids more than this price if it bids. In both examples, the uninformed buyer is more likely to bid high, given that it bids, than the informed buyer. The next two sections extend these results to the more general case where $(V, R, X)$ is affiliated.

\section{THE GENERAL MODEL}

As noted in the introduction, the evidence from the auctions of offshore oil and gas leases strongly suggests that $R$ and $V$ are positively correlated. This introduces two kinds of complications. First, it means that the informed buyer should condition his valuation of the object on the event of acceptance, since this is informative of $V$. The realization of $X$ also affects the informed buyer's estimate of the probability of acceptance. Hence, it is no longer possible to index the informed buyer types by their conditional expectation of the value of the object. The dimension of $X$ matters. As a result, we need to reformulate the maximization problems of the informed and uninformed buyers, and establish an analogue of the zero expected profit constraint. This we do in Lemma 1.

The second difficulty is also related to the dimensionality of $X$. The equilibrium analysis depends critically upon the monotonicity of the bid function. This is easily established when the information in the signal can be summarized by the conditional expected value of the object. In the general correlated case, however, it is necessary to show that $\beta$ is monotone in $X$, which is multidimensional. To ensure this result, we exploit the concept of affiliated random variables, first introduced in the bidding literature by Milgrom and Weber (1982), to impose the necessary relations between $(V, R, X)$. The relevant implications of affiliation are summarized in Lemma 2. Lemma 3 establishes the monotonicity property of $\beta$. We include its proof in the text since it is apparently the first such result that allows for a multidimensional signal.

Readers who are not interested in the technical details of the arguments underlying these results can skip to the next section, where we present the main theorems. 


\subsection{Payoffs and Equilibrium}

Let $J(\cdot \mid x)$ denote the distribution function of $R$ conditional on a realization $x$ of $X$. Then, in the absence of an uninformed buyer, $E[V-b \mid R \leqslant b, X=$ $x] J(b \mid x)$ is the expected profit to an informed buyer with information $x$ from bidding $b$. If the distribution function of the uninformed bid, $G_{\alpha}$, is continuous, then $E[V-b \mid R \leqslant b, X=x] J(b \mid x) G_{\alpha}(b)$ is the expected payoff to the informed buyer with information $x$ from bidding $b$ when facing the strategy $\alpha$.

Let $K(\cdot \mid b)$ denote the distribution function of $R$ conditional on an informed bid of $b$, induced by $J$ and $\beta$. That is, $K\left(b^{\prime} \mid b\right)$ is the probability that the reservation price is not greater than $b^{\prime}$, given that the informed bid is $b$. If the uninformed buyer wins the object with a bid of $b$, then he knows that $b$ is not less than the reservation price and that the informed bid is no greater than $b$. Both events are informative in assessing the value of the object. If $G_{\beta}$ is continuous at $b$, then $\int_{(-\infty, b]} E[V-b \mid R \leqslant b, \beta=t] K(b \mid t) P_{\beta}(d t)$ is the expected payoff to an uninformed buyer who bids $b$ when facing the strategy $\beta$.

An equilibrium is a pair $(\alpha, \beta)$ that maximizes the expected payoff to each buyer given the strategy employed by the other buyer. Since we have defined $R$ to be positive, it follows that $J(0 \mid x)=0$ a.s. $P_{x}$ so that a bid of 0 guarantees a buyer zero profits. ${ }^{5}$ Standard arguments then establish the following properties of an equilibrium.

Lemma 1: Suppose $(\alpha, \beta)$ is an equilibrium. Then, for any bid $b$ :

$$
\begin{aligned}
& E[V-\beta(x) \mid R \leqslant \beta(x), X=x] J(\beta(x) \mid x) G_{\alpha}(\beta(x)) \\
& \geqslant E[V-b \mid R \leqslant b, X=x] J(b \mid x) G_{\alpha}(b) \quad \text { a.s. } P_{x} . \\
& 0=\int_{\left(-\infty, b^{\prime}\right]} E\left[V-b^{\prime} \mid R \leqslant b^{\prime}, \beta=t\right] K\left(b^{\prime} \mid t\right) P_{\beta}(t) \\
& \geqslant \int_{(-\infty, b]} E[V-b \mid R \leqslant b, \beta=t] K(b \mid t) P_{\beta}(d t) \quad \text { a.s. } P_{\alpha} . \\
& E[V-b \mid R \leqslant b, \beta=b] K(b \mid b) P_{\alpha}(\{b\}) P_{\beta}(\{b\})=0 .
\end{aligned}
$$

Condition (c) implies that the rationing rule in the event of ties is irrelevant. The informed buyer never makes a bid which earns positive profits and has a positive probability of being matched by the uninformed buyer. By increasing his bid slightly, one of the buyers could obtain a positive gain at essentially zero cost. The inequalities in conditions (a) and (b) are then just the statement that each buyer chooses a best response given his information. Condition (b) also implies that the uninformed buyer earns zero expected profits. The argument is identical to the one we presented in the last section.

\footnotetext{
${ }^{5}$ For any random vector $Z$, let $P_{Z}$ denote the probability induced on $R^{p}$. Then, for $\alpha: R^{p} \rightarrow R$, $\alpha(z)=0$ a.s. $-P_{Z}$ if $\int|\alpha(z)| P_{Z}(d z)=0$. In general, a restriction on $Z$ is satisfied a.s. $-P_{Z}$ if it is satisfied with probability 1 with respect to $P_{Z}$.
} 
Notice that the constraint on the bid function of the informed buyer when $R$ is correlated with $(X, V)$ is that, conditional on realizations of $R$ and the bid by the informed buyer that are no greater than $b$, the expected value of the object cannot exceed $b$. The restrictions of Lemma 1 remain essentially unchanged if there is more than one uninformed buyer and $G_{\alpha}$ represents the distribution of the maximum uninformed bid. Consequently, there is no loss of generality in restricting attention to the case of a single uninformed buyer.

\subsection{Affiliation and the Monotonicity of the Informed Bid Function}

A random vector $Z$ is affiliated if any two nondecreasing functions of $Z$ are nonnegatively correlated. The precise definition and some general properties are provided in the Appendix (see also Milgrom and Weber (1982)).

Assumption 1: $(V, R, X)$ is affiliated.

Assumption 1 guarantees that the realizations of the signal can be ordered so that higher values of $X$ imply (on average) higher values of both $V$ and $R$, or at least average values which are no lower. We allow $R$ to be correlated with $V$ and, in addition, allow it to contain information about $V$ not contained in $X$. However, unless $R$ is independent of $(V, X)$ as in Section 3, risk neutrality does not imply that we can reduce $X$ to a one-dimensional signal.

Lemma 2 summarizes the critical implications of Assumption 1. The proof is presented in the Appendix.

Lemma 2: (a) $E\left[V \mid R=r^{\prime}, X=x^{\prime}\right] \leqslant E[V \mid R=r, X=x]$ for $\left(r^{\prime}, x^{\prime}\right) \leqslant(r, x)$ a.s. $P_{R \times X} \times P_{R \times X}$. (b) $J\left(r^{\prime} \mid x^{\prime}\right) J(r \mid x) \geqslant J\left(r^{\prime} \mid x\right) J\left(r \mid x^{\prime}\right)$ for $r^{\prime} \leqslant r, x^{\prime} \leqslant x$ a.s. $P_{X} \times$ $P_{X}$.

Part (a) states that the conditional expected value of the object is nondecreasing both in the reservation price and in the information of the informed buyer. If $J(\cdot \mid x)$ is differentiable with density $j(\cdot \mid x)$, part (b) is equivalent to the statement that the survival rate $j(\cdot \mid x) / J(\cdot \mid x)$ is nondecreasing in $x$. Note that, by setting $r=\infty$, part (b) also implies that $J\left(r^{\prime} \mid x\right) \leqslant J\left(r^{\prime} \mid x^{\prime}\right)$ for $x^{\prime} \leqslant x$.

To establish that the informed bid function is monotonic, we require a slight strengthening of Lemma 2(a).

Assumption 2: $E\left[V \mid R=r^{\prime}, X=x^{\prime}\right]<E[V \mid R=r, X=x]$ for $(r, x) \geqslant\left(r^{\prime}, x^{\prime}\right)$, $x \neq x^{\prime}$, a.s. $-P_{R \times X} \times P_{R \times X}$.

Assumption 2 is always satisfied for some nearby environment $\left(V^{\prime}, R, X\right)$. For instance, if $V=V^{\prime}+\varepsilon \sum_{i=1}^{n} X_{i}$, then Assumption 2 is satisfied for any $\varepsilon>0$ 
whenever $\left(V^{\prime}, R, X\right)$ satisfies Assumption $1 .^{6}$ If the reservation price is not stochastic, then, using standard arguments, Assumption 1 implies that expected profit must be nondecreasing in $x$. However, if the distribution of the reservation value shifts too much with an increase in $x$, expected profit may decline, possibly to zero. To avoid this complication and other technical points, we impose the following assumption.

Assumption 3: $J(r \mid x)>0$ implies $J\left(r \mid x^{\prime}\right)>0$ a.s. $-P_{X} \times P_{X}$.

Assumption 3 implies that the lower bound of the support of $R$ is nonnegative and independent of $X$. Our analysis requires no additional regularity conditions. Using Assumptions 1 to 3, we may establish that the equilibrium bid of the informed buyer is a nondecreasing function of the realization of $X$. The proof extends the standard self-selection argument used by EMW for the case where $R$ is constant.

Lemma 3: Suppose $(\alpha, \beta)$ is an equilibrium. Then, $E[V-\beta(x) \mid R \leqslant \beta(x), X=$ $x] J(\beta(x) \mid x) G_{\alpha}(\beta(x))>0$ implies $\beta\left(x^{\prime}\right) \geqslant \beta(x)$ for $x^{\prime} \geqslant x$, a.s. $P_{X} \times P_{X}$.

Proof: For any pair $\left(x_{1}, x_{2}\right)$, let $b_{i}=\beta\left(x_{i}\right), G_{i}=G_{\alpha}\left(b_{i}\right), J_{i j}=J\left(b_{i} \mid x_{j}\right), v_{i j}=$ $E\left[V \mid R \leqslant b_{i}, X=x_{j}\right]$, and $v_{\Delta j}=E\left[V \mid b_{1}<R \leqslant b_{2}, X=x_{j}\right], i, j=1,2$. Suppose the lemma is false. Then there is a subset $L \subset R^{n} \times R^{n}, P_{X} \times P_{X}(L)>0$, such that for $\left(x_{1}, x_{2}\right) \in L, x_{1} \geqslant x_{2}, x_{1} \neq x_{2}, b_{1}<b_{2}$, and

$$
\left(v_{22}-b_{2}\right) J_{22} G_{2}>0 \text {. }
$$

For $\left(x_{1}, x_{2}\right) \in L$, the best response property for $\beta$ (Lemma 1(a)) implies

$$
\left(v_{11}-b_{1}\right) J_{11} G_{1} \geqslant\left(v_{21}-b_{2}\right) J_{21} G_{2} \quad \text { a.s. } P_{X} \times P_{X}
$$

and

$$
\left(v_{12}-b_{1}\right) J_{12} G_{1} \leqslant\left(v_{22}-b_{2}\right) J_{22} G_{2} \quad \text { a.s. }-P_{X} \times P_{X} \text {. }
$$

Multiplying equation (4) by $J_{12}$, equation (5) by $J_{11}$, and subtracting implies that the following relation holds a.s. $-P_{X} \times P_{X}$ :

$$
\begin{aligned}
& {\left[v_{21} J_{21} G_{2}-v_{11} J_{11} G_{1}\right] J_{12}} \\
& \quad \leqslant\left[v_{22} J_{22} G_{2}-v_{12} J_{12} G_{1}\right] J_{11}+b_{2} G_{2}\left(J_{12} J_{21}-J_{11} J_{22}\right) .
\end{aligned}
$$

By definition, $v_{2 j} J_{2 j}=v_{1 j} J_{1 j}+v_{\Delta j}\left(J_{2 j}-J_{1 j}\right)$. Therefore, (6) may be written as

$$
\begin{gathered}
\left(v_{12}-v_{11}\right) J_{12} J_{11}\left(G_{2}-G_{1}\right)+\left(v_{\Delta 2}-b_{2}\right)\left(J_{11} J_{22}-J_{12} J_{21}\right) G_{2} \\
\quad+\left(v_{\Delta 2}-v_{\Delta 1}\right)\left(J_{21}-J_{11}\right) J_{12} G_{2} \geqslant 0 .
\end{gathered}
$$

\footnotetext{
${ }^{6}$ Inspection of the proof of Lemma 3 below reveals that Assumption 1 is sufficient to guarantee that a monotonic $\beta$ may be selected as a best response to $\alpha$. However, without Assumption 2, there may be another best response with a different distribution of bids in which $\beta$ is not monotonic. We have not investigated the conditions under which a continuity argument, combined with Lemma 3 below, could be used to establish the existence of an equilibrium with $\beta$ monotonic in the absence of Assumption 2.
} 
Lemma 2 and Assumptions 2 and 3 imply that all three terms in equation (7) are nonpositive. (Note that $v_{\Delta 2} \geqslant v_{22}$ by Lemma 2(a), and $v_{22}>b_{2}$ from equation (3).) All that remains to be shown is that at least one term is strictly negative. Since relation (3) implies that $J_{22}>0$, it follows from Assumption 3 that $J_{22} \geqslant J_{21}>0$. Lemma 2(a), which implies that $v_{21} \geqslant v_{22}$, and equation (3), which implies $G_{2}>0$, together with relation (4) then imply that $J_{11}>0$. Also, by definition, $J_{12}=J_{22}$ implies $v_{12}=v_{22}$. Therefore, relations (3) and (5) imply that either $G_{2}>G_{1}$ or $J_{22}>J_{12}$.

Suppose first that $G_{2}>G_{1}$. Since $J_{21}>0$ and $J_{11}>0$, and because Assumption 2 implies that $v_{12}>v_{11}$, the first term in equation (7) is then negative. Alternatively, suppose that $J_{22}>J_{12}$. Manipulation of Lemma 2(b) then implies that $J_{21}>J_{11}$, which, combined with Assumption 2, implies that the third term is negative.

Q.E.D.

To understand the role that Lemma 2 plays in the proof of Lemma 3, notice that Lemma 2(a) ensures that the first and the third terms in equation (7) are nonpositive. If $R$ is independent of $(V, X)$, then the second term of equation (7) is zero, since the distribution of $R$ does not depend upon $X$. The contradiction then follows immediately from the fact that the change in the probability of winning can be positive only if $b_{2}$ is smaller than $b_{1}$. When $(V, R, X)$ is affiliated, Lemma 2(b) ensures that the second term in equation (7) is nonpositive.

To facilitate the statement of our results, which follow in the next section, we adopt the following convention for zero profit bids.

Assumption 4: Suppose $(\alpha, \beta)$ forms an equilibrium. Then:

(a) $\int_{(-\infty, b]} K(b \mid t) P_{\beta}(d t)=0$ implies $G_{\alpha}(b)=G_{\alpha}(0)$, and

(b) $E[V-b \mid R \leqslant b, \beta=b] K(b \mid b) G_{\alpha}(b)=0$ implies $b=0$, a.s. $P_{\beta}$.

Assumption 4 requires the uninformed bidder to submit a positive bid only if he expects to win the object with positive probability. Similarly, whenever the informed buyer cannot earn positive profit, he submits a bid of zero. Given Assumption 2 and Lemma 3 , this convention guarantees that $\beta$ is nondecreasing a.s. $-P_{\beta}$, but does not restrict the equilibrium bid distributions above the lower bound of $R$.

Given that the informed bid is nondecreasing in $X$, it follows from Assumption 1 that $(V, R, \beta)$ is also affiliated. For the remainder of the analysis, we assume that $(\alpha, \beta)$ forms an equilibrium. Lemma 3 and Assumption 4 then yield the following restatement of Lemma 2 and Assumption 2 in terms of the bid of the informed buyer rather than his information signal.

LeMmA 4: (a) $E\left[V \mid R=r^{\prime}, \beta=b^{\prime}\right] \leqslant E[V \mid R=r, \beta=b],\left(r^{\prime}, b^{\prime}\right) \leqslant(r, b)$ (with strict inequality if $\left.b>b^{\prime}\right)$ a.s. $-P_{R \times \beta} \times P_{R \times \beta}$.

(b) $K\left(r^{\prime} \mid b^{\prime}\right) K(r \mid b) \geqslant K\left(r^{\prime} \mid b\right) K\left(r \mid b^{\prime}\right), r^{\prime} \leqslant r, b^{\prime} \leqslant b$ a.s. $P_{\beta} \times P_{\beta}$. 
Our results also require that the informed buyer sometimes earn strictly positive profits whenever the uninformed buyer can earn zero profits with a bid above the minimum reservation value. To ensure this property, we assume that the informed buyer sometimes bids 0 .
Assumption 5: $P_{X}\left(\left\{x \in R^{n}: J(b \mid x)>0\right.\right.$ implies $\left.\left.E[V-b \mid R \leqslant b, X=x]<0\right\}\right)>$ 0 .

Assumption 5 states that, with positive probability, the informed buyer sometimes receives a signal for which his expected profit is negative at any bid which exceeds the reservation price.

Lemma 5: (a) $P_{\beta}(\{0\})>0$, and (b) $K(b \mid 0)>0$ implies $\int E[V-b \mid R \leqslant b, \beta=$ $0] K(b \mid 0)<0$.

Proof: The result follows from Assumption 5 and Lemmata 3 and 4. Q.E.D.

The informed buyer sometimes does not bid. In those cases, his expected profit at any bid above the lowest possible reservation price is strictly negative.

\section{THE BID DISTRIBUTIONS}

In this section, we exploit the affiliation of $(V, R, \beta)$ to establish the main theoretical results of the paper. Theorem 1 establishes two results. First, the rate of increase in $\log G_{\beta}$ is never less than the rate of increase in $\log G_{\alpha}$. From this property it follows immediately that (i) the support of $\beta$ contains the support of $\alpha$, and (ii) $\beta$ stochastically dominates $\alpha$. Second, when $R$ is independent of $(V, X)$, the rate of increase in $\log G_{\beta}$ equals the rate of increase in $\log K G_{\alpha}$. It follows immediately from this property that $G_{\alpha}$ and $G_{\beta}$ are identical above the support of $R$. Theorem 2 establishes that the informed buyer is more likely to submit a low bid than is the uninformed buyer.

The details of the argument for our main result, Theorem 1, are complicated by the fact that $G_{\beta}$ need not be continuous nor strictly increasing over the convex hull of its support. Therefore, we confine the text to a heuristic demonstration of the result and provide a complete proof in the Appendix.

Theorem 1: (a) $b_{0} \leqslant b_{1}$ implies $G_{\alpha}\left(b_{0}\right) G_{\beta}\left(b_{1}\right) \geqslant G_{\alpha}\left(b_{1}\right) G_{\beta}\left(b_{0}\right)$. (b) Suppose $R$ is independent of $(V, X)$. If $G_{\alpha}$ is strictly increasing over $\left[b_{0}, b_{1}\right]$, then $K\left(b_{0}\right) G_{\alpha}\left(b_{0}\right) G_{\beta}\left(b_{1}\right)=K\left(b_{1}\right) G_{\alpha}\left(b_{1}\right) G_{\beta}\left(b_{0}\right)$.

Sketch of Proof: Suppose $G_{\alpha}$ and $G_{\beta}$ are both differentiable and increasing at bid $b>0$. Denote the densities of all distribution functions by their lower case letters and let $w=E[V \mid R \leqslant b, \beta=b], v=E[V \mid R=b, \beta=b], \bar{v}=$ $\int_{-\infty}^{b} E[V \mid R=b, \quad \beta=t] d G_{\beta}(t) / G_{\beta}(b), \quad K=K(b \mid b), \quad$ and $\bar{K}=$ $\int_{-\infty}^{b} K(b \mid t) d G_{\beta}(t) / G_{\beta}(b)$. 
Suppose the informed buyer considers a unit increase in his bid. Since he wins the object with probability $K G_{\alpha}$ at bid $b$, his expected cost increases by $K G_{\alpha}$. On the other hand, the gain in his expected profit from the additional chance of winning the object can be decomposed into two components. The first is his gain when the uninformed buyer bids just above $b,(w-b) \mathrm{Kg}_{\alpha}$. The second is his gain when the reservation price is just above $b,(v-b) k G_{\alpha}$. Consequently, the first order condition for profit maximization is

$$
(w-b) K g_{\alpha}+(v-b) k G_{\alpha}=K G_{\alpha} .
$$

Similarly, the first order condition for profit maximization by the uninformed buyer is

$$
(w-b) \bar{K} g_{\beta}+(\bar{v}-b) \bar{k} G_{\beta}=\bar{K} G_{\beta} .
$$

By Assumption 4, the informed buyer makes positive profit at bid $b$. Therefore, $w-b>0$.

To prove part (a), note first that Lemma 2(b) implies that $K \leqslant \bar{K}$, and Lemma 1(b) and Lemma 2(a) imply $\bar{v} \geqslant b$. Therefore, we may combine equations (8) and (9) to yield

$$
(w-b)\left(\frac{g_{\beta}}{G_{\beta}}-\frac{g_{\alpha}}{G_{\alpha}}\right) \geqslant(v-b) \frac{k}{K}-(\bar{v}-b) \frac{\bar{k}}{\bar{K}} .
$$

Lemma 4(a) implies $v \geqslant \bar{v}$, and Lemma 4(b) implies $k / K \geqslant \bar{k} / \bar{K}$. It follows from (10), therefore, that $g_{\beta} / G_{\beta} \geqslant g_{\alpha} / G_{\alpha}$. Integrating over $\left[b^{\prime}, b\right]$ establishes part (a).

To prove part (b), note that if $R$ is independent of $(V, X)$, then $K=\bar{K}, k=\bar{k}$, and $w=v$. Furthermore, the zero profit condition (Lemma 1(b)) implies that $\bar{v}=b$. Combining equations (9) and (10) then imply

$$
\frac{g_{\beta}}{G_{\beta}}=\frac{g_{\alpha}}{G_{\alpha}}+\frac{k}{K} .
$$

Integrating over $\left[b^{\prime}, b\right)$ establishes part (b).

Q.E.D.

Theorem 1(a) implies the following corollary.

CoRollaRy 1: (a) $G_{\alpha}\left(b_{0}\right)<G_{\alpha}\left(b_{1}\right)$ implies $G_{\beta}\left(b_{0}\right)<G_{\beta}\left(b_{1}\right)$. (b) $G_{\alpha} \geqslant G_{\beta}$.

Part (a) states that the support of $G_{\beta}$ contains the support of $G_{\alpha}$. Part (b) states that $G_{\beta}$ stochastically dominates $G_{\alpha}$. 
Notice also that, wherever $G_{\alpha}$ is increasing, Theorem 1(b) implies that the log of the distribution function of $\max (\{R, \alpha\})$ grows at the same rate as $\log G_{\beta}$. Consequently, above the support of $R, G_{\alpha}$ and $G_{\beta}$ are identical even if $(V, R, X)$ are positively correlated. Specifically, we have the following corollary.

Corollary 2: If $K(b \mid b)=1$, for $b \geqslant b_{0}$, then $G_{\alpha}(b)=G_{\beta}(b)$ for $b \geqslant b_{0}$.

Corollary 2 generalizes a result of EMW that established that $G_{\alpha}$ and $G_{\beta}$ are equal whenever the reservation price is not random.

Besides the assumption that all distribution functions are differentiable, the sketch of the proof of Theorem 1 provided above also supposes that the two distribution functions have the same support. As we saw in Example 1 of Section 4, this restriction need not be satisfied when the reservation price is random. The more general proof provided in the Appendix handles these cases as well.

We turn next to the restrictions on the distribution functions at low bids. Recall that the expected value of the object to the uninformed buyer when he wins the object with a bid of $b$ is the average value of the object to the informed buyer when his equilibrium bid is no greater than $b$. Therefore, if the informed buyer sometimes expects negative profit at any winning bid, any zero profit, positive uninformed bid must exceed some profitable informed bids. This observation motivates Theorem 2. A precise proof requires a careful limiting argument and is presented in the Appendix.

THEOREM 2: There is a $b>0$ such that $G_{\beta}(b)>G_{\beta}(0)$ and $G_{\alpha}(b)=G_{\alpha}(0)$.

Theorem 2 implies that the informed buyer is more likely to submit a low bid than is the uninformed buyer. Either the informed buyer submits his lowest bid with positive probability while the uninformed bid distribution is continuous, or there is an interval around the lowest informed bid in which the uninformed buyer never submits a bid. The size of this interval depends on the distribution of $(V, R, X)$.

\section{INFORMAL TESTS OF THE THEORY}

In this section, we analyze the bidding data from a superset of the drainage tracts used by HP in their study of auctions for offshore oil leases. Our data set contains the number and characteristics of the firms who submitted bids, the value of their bids, and the rejection decision on all drainage tracts offered for sale between 1959 and 1979 on which at least one firm submitted a bid.

As we noted in the introduction, our theoretical model is based on the analysis of HP. Working with data from tracts offered for sale during the period 1959 to 1969 , they estimated the ex post values of tracts offered for sale and the 
adjacent tracts owned by firms participating in the auctions. With these estimates, they investigated the relation between tract values and the bidding behavior of the firms. ${ }^{7}$ Their work provides evidence that neighbor firms had better information about the value of a tract than did nonneighbor firms and that the neighbor firms coordinated their bidding so that there was effectively only one informed bidder. Therefore, we ignore all but the highest neighbor bid on any tract and call it the neighbor bid, $B_{I}$. We call the highest bid among all nonneighbor firms, $B_{U}$, the high nonneighbor bid. Note that, since our theory implies that the distribution of the highest nonneighbor bids is independent of the number of nonneighbor bidders (see the end of Section 4.1), our theoretical results may be applied to auctions with more than one nonneighbor without modification. $^{8}$

Our assumption that the reserve price is an exogenous random variable is based on the HPS study of the same data set we employ here. Although the rejection decision is made after the bids are submitted, HPS found no evidence of strategic behavior on the part of the government. ${ }^{9}$ Also, based on their estimates of a probit equation of the rejection decision, they conclude that the probability of rejection was higher if the bid was submitted by a neighbor firm, even after conditioning on the value of the bid. While neighbor firms submitted the highest bid on $61 \%$ of the tracts, they submitted $79 \%$ of the rejected bids. Of tracts on which the high bid was less than .5 million dollars, 27 of the 54 bids submitted by neighbor firms were rejected while only 4 of 15 bids submitted by nonneighbor firms were rejected. ${ }^{10}$ Note that the theoretical model predicts that neighbor firms are more likely to submit low bids, and therefore more likely to submit a disproportionate fraction of rejected bids.

Any test of the theory must incorporate the fact that the properties derived in Section 4 are valid only after conditioning on all relevant information, $S$, possessed by the nonneighbor firms. Let $G_{U}(\cdot, s)$ denote the distribution function of $B_{U}$, conditional on $S=s, G_{I}(\cdot, s)$ the distribution function of $B_{I}$, conditional on $S=s$, and $\bar{r}(s)$ the upper bound of the support of $R$, conditional on $S=s$. Theorems 1 and 2 then imply the following relations for each

\footnotetext{
${ }^{7}$ For tracts sold after 1970 , they were unable to generate reliable estimates of tract value, both because the production histories are truncated and because expectations of firms about future oil and gas prices are difficult to measure or infer.

8 There were two or more bids by neighbor firms on only 59 of the 257 tracts on which at least one neighbor firm submitted a bid. There were two or more bids by nonneighbor firms on 92 of the 168 tracts on which at least one nonneighbor firm submitted a bid.

${ }^{9}$ According to Darius Gaskins (1976, p. 241), "the primary factor used in evaluating bids is the government's evaluation," which is determined prior to the sale date.

${ }^{10}$ HPS do not report the relation between the rejection decision and the number of bids. While 169 of 295 tracts received two or more bids, those tracts accounted for only 11 of the 58 rejected bids. (Of the 130 tracts that received at least one neighbor and one nonneighbor bid, only 3 were rejected.) Although these figures suggest that the number of bids may have influenced the rejection decision, HPS found that the number of bids is sufficiently correlated with the maximum bid and the neighbor dummy so that its coefficient is not significantly different from 0 in a probit analysis of the rejection decision. In any event, we have not investigated the theoretical implications of this assumption.
} 


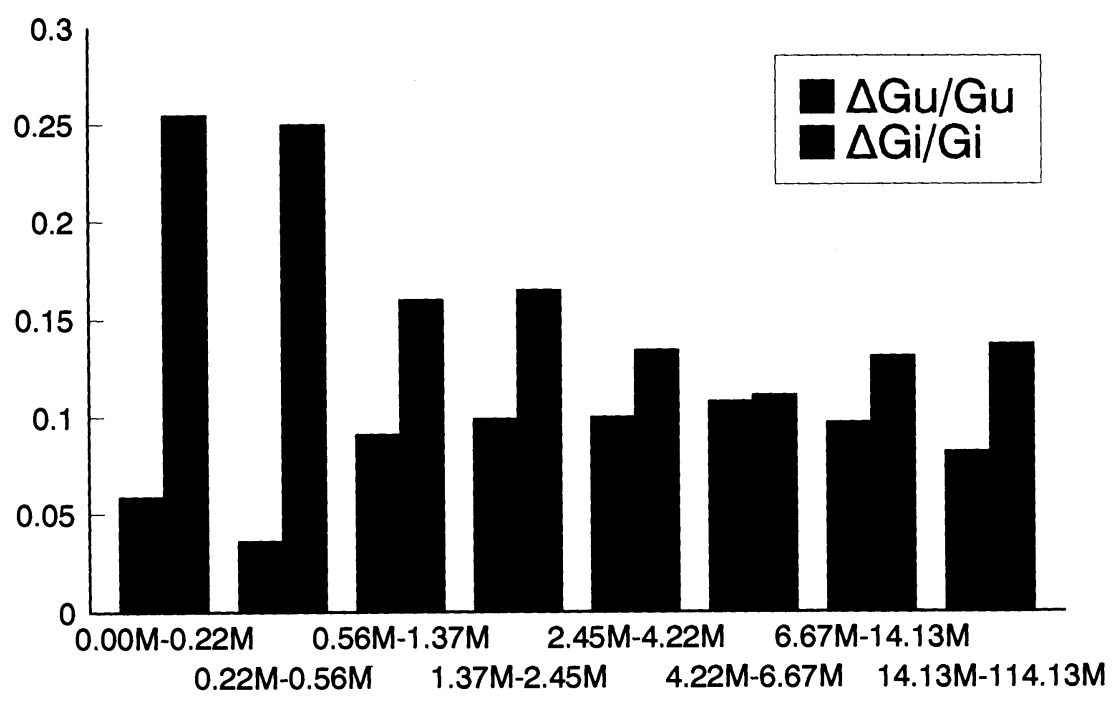

FIGURE 4.-Rates of change in the distributions.

realization $s$ of $S$ :

$$
\begin{array}{ll}
\text { (R1) }\left[G_{U}(b, s)-G_{U}(b-\varepsilon, s)\right] / G_{U}(b, s) & \\
& \leqslant\left[G_{I}(b, s)-G_{I}(b-\varepsilon, s)\right] / G_{I}(b, s) \\
& \quad \text { for } G_{I}(b, s), G_{U}(b, s)>0 \quad \text { and } \varepsilon>0 . \\
\text { (R2) } \quad & G_{U}(b, s)=G_{I}(b, s), \quad b \geqslant \bar{r}(s) . \\
\text { (R3) } \quad \text { For some } \underline{b}(s), \quad G_{I}(\underline{b}(s), s)>G_{I}(0, s) \quad \text { and } \\
& G_{U}(\underline{b}(s), s)=G_{U}(0, s) .
\end{array}
$$

Ignoring for the moment the complications introduced by the information variable $S$, the histograms presented in Figures 4 and 5 illustrate two aspects of the empirical distributions which directly test these relations. Figure 4 illustrates the relation between $\Delta G_{I} / G_{I}$ and $\Delta G_{U} / G_{U}$. The ratios were constructed by partitioning the set of all positive neighbor and high nonneighbor bids into 8 equally sized subsets according to their rank. The interval of bids for each subset is indicated on the horizontal axis. For each interval of bids we then divided the number of neighbor bids in that interval by the number of tracts for which the neighbor bid was in that interval or below plus the number of tracts for which no neighbor firm submitted a bid to obtain $\Delta G_{I} / G_{I}$. We computed $\Delta G_{U} / G_{U}$ similarly.

Figure 4 lends strong support to all three relations. First, $\Delta G_{I} / G_{I}$ is as least as large as $\Delta G_{U} / G_{U}$ over each range of bids, as required by (R1). Second, the two ratios are roughly equal in the upper four intervals as is required if the distributions are equal over that range. Finally, $\Delta G_{I} / G_{I}$ exceeds $\Delta G_{U} / G_{U}$ by more than a factor of 5 over the first two intervals, lending support to (R3). 


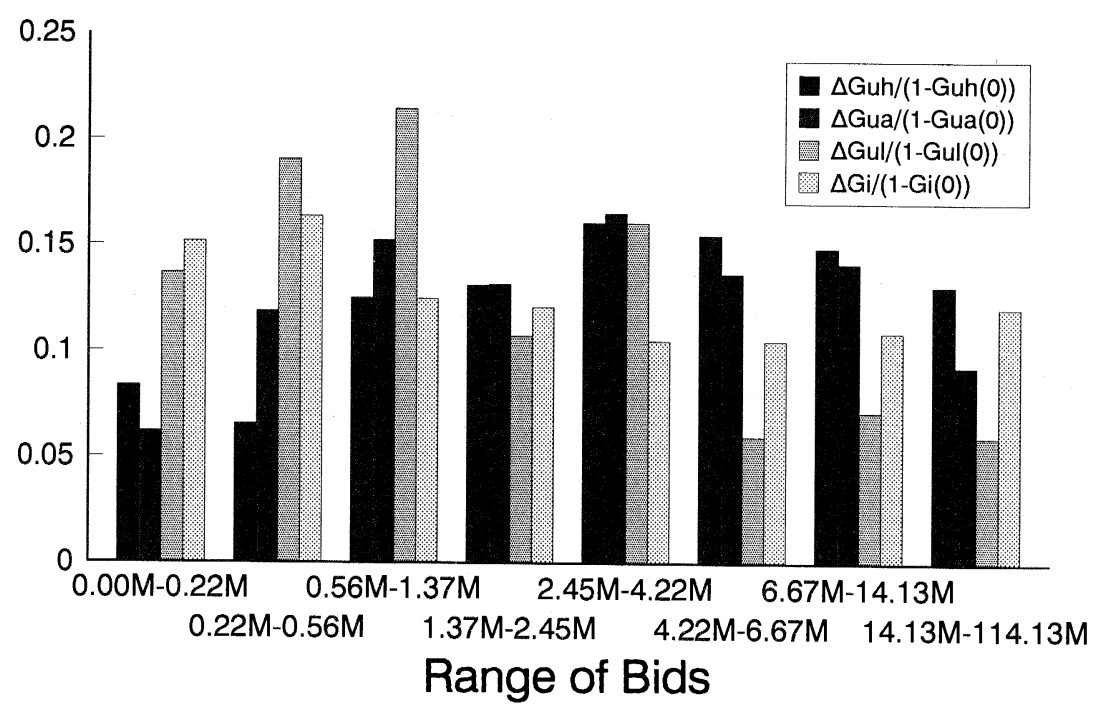

Figure 5.-Relative number of low bids.

Figure 5 provides additional evidence regarding the relative frequency of low bids by the neighbor and nonneighbor firms. Relation (R3) implies that over intervals of low bids, there should be relatively more high neighbor bids than high nonneighbor bids. In fact, regardless of which nonneighbor bid is selected on any tract, the theory implies that there should be more neighbor than (high) nonneighbor bids. Figure 5 illustrates the ratio of the number of bids in any interval to the total number of positive bids for the following four criteria: (i) the high positive nonneighbor bid on each tract (with distribution function $G_{I}$ ), (ii) all positive nonneighbor bids $\left(G_{U A}\right)$, (iii) the low positive nonneighbor bid on each tract $\left(G_{U L}\right)$, and (iv) all positive (high) neighbor bids $\left(G_{I U H} ; G_{U}\right.$ in Figure 4). Notice that in the lowest interval, the relative number of neighbor bids is twice the relative number both of high nonneighbor bids and of all nonneighbor bids. The relative number of low nonneighbor bids in this interval is roughly equal to the relative number of neighbor bids. Although a bit weaker, these relations are also satisfied in the second interval. We note also that since relatively more tracts received at least one positive bid by a neighbor firm, these figures actually understate the predominance of neighbor bids in the lower interval when we include tracts with no bids by nonneighbor firms.

Figure 1 summarizes some of the information depicted in Figures 4 and 5. It illustrates the greater propensity of neighbor firms to submit a bid and the greater likelihood of neighbor firms to submit low bids when they participate. Moreover, the distribution function of the high neighbor bid stochastically dominates that of the high nonneighbor bid with a rough coincidence of the bid distributions above 4 million dollars. Therefore, our data set, as depicted in Figures 1,4 , and 5 , is suggestive of the validity of the theory. 
Since HP report that most tracts are about 2,500 acres with an official announced bid of $\$ 25 /$ acre, it may be reasonable to suppose that the distribution of the reserve price has a probability mass at $\$ 62,500$. If so, reasonable restrictions on the distribution of information of the informed bidders imply a mass point in the distribution of the informed bid at that price. ${ }^{11}$ Although we believe that $\$ 62,500$ is a good estimate of the lower bound of the distribution of $R$, we are less convinced that the buyers perceive a mass point at $\$ 62,500$. As noted in the introduction, roughly $20 \%$ of the auctions resulted in the rejection of bids at higher prices. In any event, the neighbor firms do not appear to have bid near $\$ 62,500$ with significant probability. (Some care must be taken in the interpretation of Figure 1, however, since those figures are all translated to 1972 prices.)

Without making parametric assumptions, a rigorous test of relations (R1) to (R3) is not possible because of two difficulties. First, the data are drawn from a truncated distribution, in that our data include only tracts that received at least one bid. In particular, this presents a problem for tests of relation (R1). Second, the distributions of $B_{I}$ and $B_{U}$ are almost surely not independent, and much of the heterogeneity across tracts in publicly available information variables is not observable by the econometrician. ${ }^{12}$

Our 1990 working paper employs nonparametric tests that apply the Wilcoxon rank sum statistic. These tests indicate that $B_{I}$ and $B_{U}$ are not independently distributed in our sample. After accounting for unobservable heterogeneity across tracts, it still appears that the relations illustrated in Figures 1, 4, and 5 are statistically significant. However, these nonparametric tests are not definitive, but merely suggestive.

To estimate a parametric specification, it may be necessary to employ numerical methods in both estimation and simulation of a structural econometric model. There is no readily available affiliated joint distribution of $(R, X, V)$ that yields closed form bid functions. However, one might assume that $(R, X, V)$ are drawn from a multivariate lognormal distribution, which is affiliated if covariances are nonnegative, and characterize the first order conditions for equilibrium bidding strategies. Then simulated moments estimation methods could be used to recover the parameters of the joint distribution, and overidentifying restrictions could be tested. Analogous methods are described by Laffont, Ossard, and Vuong (1993), in the case of symmetric independent private value auctions with a known reserve price.

${ }^{11}$ Let $r$ be the greatest lower bound of the distribution of $R$ and suppose that $J(r, X=x)>0$ for all $x$. If, in addition to Assumptions 1-5, we also assume that $\operatorname{Pr}(E[V \mid X]) \in(\underline{r}, \underline{r}+\varepsilon)>0$ for any $\varepsilon>0$, then it may be shown that the distribution of the informed bid must have a mass point at $r$.

${ }^{\overline{1} 2}$ In their earlier study, HP established that, conditioning on the number of neighbor firms, the tract acreage, and the ex post value of the adjacent tract (the most significant of these variables), there is no significant relation between the high nonneighbor bid and the ex post value of the tract. From this, they conclude that these variables contain most of the information possessed by nonneighbor firms in assessing the value of the tract. As noted above, however, we are unable to construct this information for most of the tracts in our sample. 
In any case, reduced form tests of the theory are sufficient for our purposes. Knowledge of the structural parameters of the joint distribution of $(V, R, X)$ is not intrinsically important for mechanism design (see, for example, Hendricks, Porter, and Tan (1993)), as opposed to knowing whether the predictions of the theory match the data. If the economic environment can be characterized by asymmetric information, a random reserve price, and approximately risk neutral, Bayesian Nash equilibrium behavior, then there is sufficient information to assess the institution. Of course, structural estimation would be useful to the extent that overidentifying assumptions implied by the theory can be tested.

\section{CONCLUSION}

This paper examines how asymmetries in the distribution of information among agents affect their behavior in a strategic setting. We study this issue in the context of a first-price auction with a random reservation price, in which one buyer has superior private information and all other buyers have access only to public information. Equilibrium bidding behavior under this information structure requires that uninformed buyers collectively bid less frequently than the informed buyer but, if they bid, they submit high rather than low bids. Specifically, we show that the distribution of the informed bid stochastically dominates the distribution of the high uninformed bid in the support of the reservation price, and the two distributions are identical above this range. These theoretical implications are strongly borne out by our data from the auctions for offshore oil drainage leases.

Our empirical work suffers from the absence of an alternative model. It is difficult to construct alternative models that fit the data as well. For example, one might hypothesize that neighbor firms place a higher value on deposits on adjacent tracts than nonneighbor firms because they can internalize possible production externalities associated with a common pool. This could explain why nonneighbor firms are less likely to bid than neighbor firms and some aspects of the stochastic dominance relation between the distributions of the high nonneighbor and neighbor bids. However, it fails to explain the equivalence of the distributions of the high neighbor and nonneighbor bids above the support of the reservation price. To explain this feature of the data, one needs to assume that the production externality is present on small pools only. In that case, the neighbor firm may bid low more frequently than nonneighbor firms, since the latter may want to bid only on tracts that are likely to possess large pools. Such an assumption does not appear very plausible. It also fails to explain the relatively weak correlation between lease value and nonneighbor participation and bids.

We have not addressed the issue of the optimality of the random reservation price rule used by the federal government, focussing instead on its implications for optimal bidding. Recent work by McAfee and Vincent (1992) suggests that current practice is not optimal. However, they do not contrast fixed and random reservation price strategies, and in any case, it is debatable whether their 
evidence is conclusive. Clearly, more work needs to be done on this subject. As Ashenfelter's (1989) discussion of wine auctions demonstrates, a random reservation price strategy is not unique to offshore oil and gas lease auctions, but can also be found in the private sector. Therefore, the optimality and implications of different reservation price strategies for bidding behavior may be of more general interest than suggested by our work here.

Department of Economics, University of British Columbia, 997-1873 East Mall, Vancouver, BC V6T 1Z1, Canada,

Department of Economics, Northwestern University, 2003 Sheridan Rd., Evanston, IL 60208, U.S.A.,

and

Department of Economics, New York University, 269 Mercer St., New York, NY 10003, U.S.A.

Manuscript received August, 1991; final revision received March, 1994.

\section{APPENDIX}

A1. THE EFFECT OF INTRODUCING UNINFORMED BUYERS ON THE INFORMED BID

In this section, we establish that the introduction of uninformed buyers never lowers the equilibrium bid of the informed buyer. We suppose that bids must be nonnegative.

THEOREM A1: If the auction with no uninformed buyers has an equilibrium, then it has an equilibrium $\beta^{0}$ such that, for any equilibrium $(\beta, \alpha)$ with an uninformed buyer, $G_{\alpha}(\beta(x))>0$ implies $\beta^{0}(x) \leqslant \beta(x)$ a.s. $P_{X}$.

Proof: Let $(\beta, \alpha)$ be an equilibrium for an auction with an uninformed buyer and $\beta^{0}$ an equilibrium for an auction with no uninformed buyer. Then, since $E[V-b \mid R \leqslant b, X=x] J(b \mid x)$ is right continuous in $b$ a.s. $P_{X}$, we may define $\beta^{0}(x)$ to be the smallest maximizer of $E[V-b \mid R \leqslant b$, $X=x] J(b \mid x)$ a.s. $P_{X}$. Let $L=\left\{x \in R^{p}: \beta(x)<\beta^{0}(x)\right.$ and $\left.G_{\alpha}(\beta(x))>0\right\}$. Suppose $P_{X}(L)>0$. Then, the definition of $\beta^{0}$ implies

$$
\begin{aligned}
\int_{\{x \in L\}} E[V-\beta(X) \mid R \leqslant \beta(x), X=x] J(\beta(x) \mid x) G_{\alpha}(\beta(x)) P_{X}(d x) \\
\quad<\int_{\{x \in L\}} E\left[V-b \mid R \leqslant \beta^{0}(x), X=x\right] J\left(\beta^{0}(x) \mid x\right) G_{\alpha}(\beta(x)) P_{X}(d x) .
\end{aligned}
$$

But since $G_{\alpha}(\beta(x)) \leqslant G_{\alpha}\left(\beta^{0}(x)\right)$ for $x \in L$, the best response property for $\beta$ (Lemma 1(a)) requires

$$
\begin{aligned}
\int_{\{x \in L\}} E[V-\beta(X) \mid R \leqslant \beta(x), X=x] J(\beta(x) \mid x) G_{\alpha}(\beta(x)) P_{X}(d x) \\
\geqslant \int_{\{x \in L\}} E\left[V-\beta^{0}(X) \mid R \leqslant \beta^{0}(x), X=x\right] J\left(\beta^{0}(x) \mid x\right) G_{\alpha}\left(\beta^{0}(x)\right) P_{X}(d x) \\
\geqslant \int_{\{x \in L\}} E\left[V-\beta^{0}(X) \mid R \leqslant \beta^{0}(x), X=x\right] J\left(\beta^{0}(x) \mid x\right) G_{\alpha}(\beta(x)) P_{X}(d x),
\end{aligned}
$$

a contradiction. 


\section{A2. AFFiLIATION}

In this section, we define the concept of affiliated random variables and establish Lemma 2 of the text. If $Z$ is a random vector taking values in $R^{p}$, then the probability measure on $R^{p}$ induced by $Z$ is denoted by $P_{Z}$.

For $x, y \in R^{p}$, let $x \wedge y$ denote the pointwise minimum of $x$ and $y$, and let $x \vee y$ denote the pointwise maximum of $x$ and $y$. A function $f: R^{p} \rightarrow R$ is affiliated if $x, y \in R^{p}$ implies

$$
f(x \wedge y) f(x \vee y) \geqslant f(x) f(y) .
$$

A set $S \in R^{p}$ is a sublattice if $\iota_{S}$ is affiliated.

For any set $A \subset R^{p}$, let $\iota_{A}$ denote the indicator function for $A$ on $R^{p}$. A set $A \in R^{p}$ is increasing if $\iota_{A}$ is nondecreasing. Given two sets $A$ and $B$, let $A B$ denote their intersection. A random variable $Z$ is affiliated if, for all increasing subsets $A_{1}, A_{2}$ of $R^{p}$ and every sublattice $S$ of $R^{p}$, $P_{Z}\left(A_{1} A_{2} S\right) P_{Z}(S) \geqslant P_{Z}\left(A_{1} S\right) P_{Z}\left(A_{2} S\right)$. Milgrom and Weber (1982) establish the following result.

LEMMA A1: $Z$ is affiliated if and only if for any nondecreasing functions $\alpha_{1}$ and $\alpha_{2}$ on a sublattice $S \subset R^{p}, E\left[\alpha_{1}(Z) \alpha_{2}(Z) \iota_{S}(Z)\right] E\left[\iota_{S}(Z)\right] \geqslant E\left[\alpha_{1}(Z) \iota_{s}(Z)\right] E\left[\alpha_{2}(Z) \iota_{S}(Z)\right]$.

Proof of Lemma 2: (a) Let $I$ and $J$ be two disjoint intervals ${ }^{13}$ of $R \times R^{n}$ such that $\left(x^{\prime}, r^{\prime}\right) \in I$ and $(x, r) \in J$ implies $x^{\prime} \leqslant x$ and $r^{\prime} \leqslant r$. Let $T$ be the minimal sublattice containing $I \cup J$, let $S=R \times T$, and let $Z=(V, R, X)$. Then, since $T$ is a lattice, letting $\alpha_{1}(Z)=V$, and $\alpha_{2}(Z)=\iota_{J}(R, X)$, Assumption 1 and Lemma A1 imply that

$$
E\left[V \iota_{J}(R, X)\right] E\left[\iota_{T}(R, X)\right] \geqslant E\left[V \iota_{T}(R, X)\right] E\left[\iota_{J}(R, X)\right] .
$$

Similarly, letting $\alpha_{1}(Z)=V$ and $\alpha_{2}(Z)=\iota_{(T-I)}(X)$, the application of Lemma A1 implies, after cancelling terms,

$$
E\left[V \iota_{T}(R, X)\right] E\left[\iota_{I}(R, X)\right] \geqslant E\left[V \iota_{I}(R, X)\right] E\left[\iota_{T}(R, X)\right] .
$$

Multiplying (A1) and (A2) and cancelling terms, then yields

$$
E\left[V \iota_{J}(R, X)\right] E\left[\iota_{I}(R, X)\right] \geqslant E\left[\iota_{J}(R, X)\right] E\left[V \iota_{I}(R, X)\right] .
$$

(b) Let $I$ and $J$ be two disjoint intervals of $R^{n}$ such that $x^{\prime} \in I$ and $x \in J$ implies $x^{\prime} \leqslant x$ and let $T$ be the minimal sublattice containing $I \cup J$. Let $S=R \times(-\infty, r] \times T, Z=(V, R, X)$, and $\alpha_{1}(Z)=$ $\iota_{\left(r^{\prime}, r\right]}(R)$. Suppose first that $\alpha_{2}(Z)=\iota_{J}(X)$. Then Assumption 1 and Lemma A1 imply

$$
\begin{aligned}
& E\left[\iota_{\left.r^{\prime}, r\right]}(R) \iota_{J}(X)\right] E\left[\iota_{(-\infty, r]}(R) \iota_{T}(X)\right] \\
& \quad \geqslant E\left[\iota_{\left(r^{\prime}, r\right]}(R) \iota_{S}(X)\right] E\left[\iota_{(-\infty, r]}(R) \iota_{J}(X)\right] .
\end{aligned}
$$

Suppose next that $\alpha_{2}(Z)=-\iota_{I}(X)$. Then a similar application of Lemma A1 implies, after cancelling terms,

$$
\begin{aligned}
& E\left[\iota_{\left(r^{\prime}, r\right]}(R) \iota_{T}(X)\right] E\left[\iota_{(-\infty, r]}(R) \iota_{I}(X)\right], \\
& \quad \geqslant E\left[\iota_{\left(r^{\prime}, r\right]}(R) \iota_{I}(X)\right] E\left[\iota_{(-\infty, r]}(R) \iota_{T}(X)\right] .
\end{aligned}
$$

Multiplying (A4) and (A5) and simplifying yields

$$
\begin{aligned}
& E\left[\iota_{\left(-\infty, r^{\prime}\right]}(R) \iota_{I}(X)\right] E\left[\iota_{\left(-\infty, r^{\prime}\right]}(R) \iota_{J}(X)\right] \\
& \quad \leqslant E\left[\iota_{\left(-\infty, r^{\prime}\right]}(R) \iota_{J}(X)\right] E\left[\iota_{(-\infty, r]}(R) \iota_{I}(X)\right] .
\end{aligned}
$$
(A6).

Since the intervals of $R^{n}$ generate its Borel sets, parts (a) and (b) follow from relations (A3) and

${ }^{13}$ A subset $A$ is an interval of a subset $S \subset R^{p}$ if there are $p$-tuples $\left(a_{1}, \ldots, a_{p}\right)$ and $\left(b_{1}, \ldots, b_{p}\right)$ such that $A=\left\{x \in S: a_{i} \leqslant(<) x_{i} \leqslant(<) b_{i}, i=1, \ldots, p\right\}$, where the symbol $\leqslant(<)$ indicates that we are allowing arbitrary open, half-open, and closed intervals. 
A3. PROOFS OF THEOREMS 1 AND 2

If $f$ is a monotonic function, let $f^{-}(r)=\lim _{s \uparrow r} f(s)$.

LEMMA A2: Suppose $f$ and $g$ are nondecreasing, nonnegative, right continuous functions. Then $f\left(r_{0}\right) g\left(r_{1}\right)<f\left(r_{1}\right) g\left(r_{0}\right), r_{0}<r_{1}$ implies an $\eta<1$ and $r_{2}>r_{0}$ such that $\left[g^{-}\left(r_{2}\right)-g(r)\right] f(r)<\eta\left[f\left(r_{2}\right)\right.$ $-f(r)] g(r), r \in\left(r_{0}, r_{2}\right)$.

PRoof: Suppose $f\left(r_{0}\right) g\left(r_{1}\right)<f\left(r_{1}\right) g\left(r_{0}\right)$ for some $r_{0}<r_{1}$. Then we may choose $\eta_{0}<1$ so that $\left[g\left(r_{1}\right)-\eta_{0} g\left(r_{0}\right)\right] /\left[f\left(r_{1}\right)-f\left(r_{0}\right)\right]<\eta_{0} g\left(r_{0}\right) / f\left(r_{0}\right)$. Since $\eta_{0}<1$ and $f$ and $g$ are right continuous and nondecreasing, $\left[g^{-}(r)-\eta_{0} g\left(r_{0}\right)\right] /\left[f(r)-f\left(r_{0}\right)\right]$ attains a minimum over $\left(r_{0}, r_{1}\right]$ at some $r_{2}$ for which $f(r)<f\left(r_{2}\right)$ for $r<r_{2}$. Therefore, for $r \in\left(r_{0}, r_{2}\right)$,

$$
\begin{aligned}
{\left[g^{-}(r)-\eta_{0} g\left(r_{0}\right)\right]\left[f\left(r_{2}\right)-f\left(r_{0}\right)\right] \geqslant } & {\left[g^{-}\left(r_{2}\right)-\eta_{0} g\left(r_{0}\right)\right]\left[f(r)-f\left(r_{0}\right)\right] } \\
= & {\left[g^{-}\left(r_{2}\right)-\eta_{0} g\left(r_{0}\right)\right]\left[f\left(r_{2}\right)-f\left(r_{0}\right)\right] } \\
& -\left[g^{-}\left(r_{2}\right)-\eta_{0} g\left(r_{0}\right)\right]\left[f\left(r_{2}\right)-f(r)\right]
\end{aligned}
$$

which implies

$$
\left[g^{-}\left(r_{2}\right)-\eta_{0} g\left(r_{0}\right)\right]\left[f\left(r_{2}\right)-f(r)\right] \geqslant\left[g^{-}\left(r_{2}\right)-g(r)\right]\left[f\left(r_{2}\right)-f\left(r_{0}\right)\right] .
$$

By definition, $\left[g^{-}\left(r_{2}\right)-\eta_{0} g\left(r_{0}\right)\right] /\left[f\left(r_{2}\right)-f\left(r_{0}\right)\right]<\eta_{0} g\left(r_{0}\right) / f\left(r_{0}\right)$. Multiplying both sides by $\left[f\left(r_{2}\right)-f\left(r_{0}\right)\right] f\left(r_{0}\right)$, adding $g^{-}\left(r_{2}\right) f\left(r_{2}\right)+\eta_{0} g\left(r_{0}\right) f\left(r_{0}\right)$, and simplifying implies an $\eta<1$ such that

$$
\left[g^{-}\left(r_{2}\right)-\eta_{0} g\left(r_{0}\right)\right] f\left(r_{2}\right)<\eta g^{-}\left(r_{2}\right)\left[f\left(r_{2}\right)-f\left(r_{0}\right)\right]
$$

Multiplying relation (A7) by $f\left(r_{2}\right)$, substituting relation (A8), and dividing by $\left[f\left(r_{2}\right)-f\left(r_{0}\right)\right]$ then yields $\eta g^{-}\left(r_{2}\right)\left[f\left(r_{2}\right)-f(r)\right]>f\left(r_{2}\right)\left[g^{-}\left(r_{2}\right)-g(r)\right]$ which may be rewritten as

$$
\begin{aligned}
(\eta-1) & {\left[g^{-}\left(r_{2}\right)-g(r)\right]\left[f\left(r_{2}\right)-f(r)\right]+\eta g(r)\left[f\left(r_{2}\right)-f(r)\right] } \\
> & f(r)\left[g^{-}\left(r_{2}\right)-g(r)\right] .
\end{aligned}
$$

LeMmA A3: $G_{\alpha}\left(b_{0}\right)<G_{\alpha}\left(b_{1}\right)$ implies $G_{\beta}\left(b_{0}\right)<G_{\beta}\left(b_{1}\right)$.

Proof: Suppose not. Then $b_{1}$ may be chosen so that $b<b_{1}$ implies $G_{\alpha}(b)<G_{\alpha}\left(b_{1}\right)$. Then, since $G_{\beta}\left(b_{0}\right)=G_{\beta}\left(b_{1}\right)$, the best response property for the uninformed buyer (Lemma $1(\mathrm{~b})$ ) then implies that

$$
\begin{aligned}
0 & =\int_{\left(-\infty, b_{0}\right]} E\left[V-b_{1} \mid R \leqslant b_{1}, \beta=b\right] K\left(b_{1} \mid b\right) P_{\beta}(d b) \\
& \geqslant \int_{\left(-\infty, b_{0}\right]} E\left[V-b_{0} \mid R \leqslant b_{0}, \beta=b\right] K\left(b_{0} \mid b\right) P_{\beta}(d b) .
\end{aligned}
$$

Our convention that positive bids must win the object with positive probability (Assumption 4) implies that $K\left(b_{1} \mid b_{0}\right) G_{\beta}\left(b_{0}\right)>0$. Therefore, $b_{0}$ may be chosen so that $b<b_{0}$ implies $G_{\beta}(b)<$ $G_{\beta}\left(b_{0}\right)=G_{\beta}\left(b_{1}\right)$. Since the informed buyer sometimes earns negative profits at $b_{0}$ (Lemma 5), (A9) implies that $E\left[V-b_{1} \mid R \leqslant b_{1}, \beta=b_{0}\right] K\left(b_{1} \mid b_{0}\right)>0$. The best response property for informed buyers (Lemma $1(\mathrm{a})$ ) and the monotonicity of $G_{\alpha}$ then imply that

$$
E\left[V-b_{0} \mid R \leqslant b_{0}, \beta=b_{0}\right] K\left(b_{0} \mid b_{0}\right)>E\left[V-b_{1} \mid R \leqslant b_{1}, \beta=b_{0}\right] K\left(b_{1} \mid b_{0}\right)>0 .
$$


We will show that relations (A9) and (A10) are inconsistent. Note first that Lemma 4(a) and relation (A9) imply

$$
\begin{aligned}
& E\left[V-b_{1} \mid b_{0} \leqslant R \leqslant b_{1}, \beta=b_{0}\right] \int\left[K\left(b_{1} \mid b\right)-K\left(b_{0} \mid b\right)\right] P_{\beta}(d b) \\
& \quad \geqslant \int E\left[V-b_{1} \mid b_{0} \leqslant R \leqslant b_{1}, \beta=b\right]\left[K\left(b_{1} \mid b\right)-K\left(b_{0} \mid b\right)\right] P_{\beta}(d b) \\
& \quad \geqslant\left[b_{1}-b_{0}\right] \int K\left(b_{0} \mid b\right) P_{\beta}(d b) .
\end{aligned}
$$

Multiplying by $K\left(b_{0} \mid b_{0}\right)$ then implies

$$
\begin{gathered}
E\left[V-b_{1} \mid b_{0} \leqslant R \leqslant b_{1}, \beta=b_{0}\right] K\left(b_{0} \mid b_{0}\right) \int\left[K\left(b_{1} \mid b\right)-K\left(b_{0} \mid b\right)\right] P_{\beta}(d b) \\
\geqslant\left[b_{1}-b_{0}\right] K\left(b_{0} \mid b_{0}\right) \int K\left(b_{0} \mid b\right) P_{\beta}(d b) .
\end{gathered}
$$

But Lemma 4(b) implies that $\left[K\left(b_{1} \mid b\right)-K\left(b_{0} \mid b\right)\right] K\left(b_{0} \mid b_{0}\right) \leqslant\left[K\left(b_{1} \mid b_{0}\right)-K\left(b_{0} \mid b_{0}\right)\right] K\left(b_{0} \mid b\right)$, a.s.$P_{\beta}$. Substituting into the left side of $(\mathrm{A} 11)$ and dividing by $\int K\left(b_{0} \mid b\right) P_{\beta}(d b)$ then yields

$$
E\left[V-b_{1} \mid b_{0} \leqslant R \leqslant b_{1}, \beta=b_{0}\right]\left[K\left(b_{1} \mid b_{0}\right)-K\left(b_{0} \mid b_{0}\right)\right] \geqslant\left[b_{1}-b_{0}\right] K\left(b_{0} \mid b_{0}\right)
$$

which violates relation (A10).

Q.E.D.

Proof of Theorem 1: Suppose part (a) is false. Then $G_{\alpha}\left(b_{0}\right) G_{\beta}\left(b_{1}\right)<G_{\alpha}\left(b_{1}\right) G_{\beta}\left(b_{0}\right)$ for some $b_{0}<b_{1}$. Since $G_{\alpha}$ and $G_{\beta}$ are right continuous, it follows from Lemma A2 that $b_{1}$ may be chosen so that, for some $\eta<1$,

$$
\left[G_{\beta}^{-}\left(b_{1}\right)-G_{\beta}(b)\right] G_{\alpha}(b)<\eta\left[G_{\alpha}\left(b_{1}\right)-G_{\alpha}(b)\right] G_{\beta}(b), \quad b \in\left(b_{0}, b_{1}\right) .
$$

We will use the best response properties of Lemma 1 to establish a contradiction to (A12).

Since (A12) implies that $G_{\alpha}(b)<G_{\alpha}\left(b_{1}\right)$ for $b<b_{1}$, the zero profit condition for the uninformed buyer (Lemma 1(b)) implies a nondecreasing sequence $b^{k} \uparrow b_{1}$ such that

$$
\int_{\left(-\infty, b^{k}\right]} E\left[V-b^{k} \mid R \leqslant b^{k}, \beta=b\right] K\left(b^{k} \mid b\right) P_{\beta}(d b)=0, \quad k=1,2, \ldots
$$

We show first that $P_{\beta}\left(\left\{b_{1}\right\}\right)=0$. Suppose not. Then, (A13) and Assumption 5 imply that $E\left[V-b_{1} \mid R \leqslant b_{1}, \beta=b_{1}\right] K\left(b_{1} \mid b_{1}\right)>0$. But relation (A13) also implies that

$$
\begin{aligned}
E[V- & \left.b_{1} \mid R \leqslant b_{1}, \beta=b_{1}\right] K\left(b_{1} \mid b_{1}\right) P_{\beta}\left(\left\{b_{1}\right\}\right) \\
= & \int_{\left(-\infty, b_{1}\right]} E\left[V-b_{1} \mid R \leqslant b_{1}, \beta=b\right] K\left(b_{1} \mid b\right) P_{\beta}(d b) \\
& \quad-\lim _{k \rightarrow \infty} \int_{\left(-\infty, b^{k}\right]} E\left[V-b^{k} \mid R \leqslant b^{k}, \beta=b\right] K\left(b^{k} \mid b\right) P_{\beta}(d b) \\
= & 0 .
\end{aligned}
$$

We conclude that $G_{\beta}^{-}\left(b_{1}\right)=G_{\beta}\left(b_{1}\right)$ so that (A12) may be written as

$$
\left[G_{\beta}\left(b_{1}\right)-G_{\beta}(b)\right] G_{\alpha}(b)<\eta\left[G_{\alpha}\left(b_{1}\right)-G_{\alpha}(b)\right] G_{\beta}(b), \quad b \in\left(b_{0}, b_{1}\right) .
$$

Relation (A14) and Lemma A3 imply that $G_{\beta}(b)<G_{\beta}\left(b_{1}\right), b<b_{1}$. Therefore, (A13) and our convention that positive bids must earn positive profits (Assumption 4) implies that $\left(b^{k}\right.$ ) may be chosen so that $\int_{\left(-\infty, b^{k}\right]} E\left[V-b^{k} \mid R \leqslant b^{k}, \beta=b^{k}\right] K\left(b^{k} \mid b^{k}\right)>0, k=1,2, \ldots$ Therefore, since $\eta<1$ and $P_{\beta}\left(\left\{b_{1}\right\}\right)=0$, it follows from Lemma 4 that $b_{0}$ may be chosen so that

$$
\begin{aligned}
& E\left[V-b_{1} \mid R \leqslant b_{1}, \beta=b_{0}\right] K\left(b_{1} \mid b_{0}\right)\left[G_{\beta}\left(b_{1}\right)-G_{\beta}\left(b_{0}\right)\right] \\
& \quad \geqslant \eta \int_{\left(b_{0}, b_{1}\right]} E\left[V-b_{1} \mid R \leqslant b_{1}, \beta=b\right] K\left(b_{1} \mid b\right) P_{\beta}(d b)>0 .
\end{aligned}
$$


The best response properties (Lemma 1) also imply that $b_{0}$ may be chosen to satisfy the following inequalities:

$$
\begin{aligned}
& E\left[V-b_{0} \mid R \leqslant b_{0}, \beta=b_{0}\right] K\left(b_{0} \mid b_{0}\right) G_{\alpha}\left(b_{0}\right) \\
& \quad \geqslant E\left[V-b_{1} \mid R \leqslant b_{1}, \beta=b_{0}\right] K\left(b_{1} \mid b_{0}\right) G_{\alpha}\left(b_{1}\right)
\end{aligned}
$$

and

$$
\begin{aligned}
& \int_{\left(-\infty, b_{1}\right]} E\left[V-b_{1} \mid R \leqslant b_{1}, \beta=b\right] K\left(b_{1} \mid b\right) P_{\beta}(d b) \\
& \geqslant \int_{\left(-\infty, b_{0}\right]} E\left[V-b_{0} \mid R \leqslant b_{0}, \beta=b\right] K\left(b_{0} \mid b\right) P_{\beta}(d b) .
\end{aligned}
$$

For $b \geqslant 0$ and $i=1,2$, let $K_{i}(b)=K\left(b_{i} \mid b\right), w_{i}(b)=E\left[V \mid R \leqslant b_{i}, \beta=b\right]$, and $w_{\Delta}(b)=E\left[V \mid b_{0}<\right.$ $\left.R \leqslant b_{1}, \beta=b\right]$. Then relation (A16) implies

$$
\begin{aligned}
& {\left[w_{1}\left(b_{0}\right)-b_{1}\right] K_{1}\left(b_{0}\right)\left[G_{\alpha}\left(b_{1}\right)-G_{\alpha}\left(b_{0}\right)\right]} \\
& \quad+\left[\left[w_{\Delta}\left(b_{0}\right)-b_{0}\right]\left[K_{1}\left(b_{0}\right)-K_{0}\left(b_{0}\right)\right]-\left[b_{1}-b_{0}\right] K_{1}\left(b_{0}\right)\right] G_{\alpha}\left(b_{0}\right) \leqslant 0 .
\end{aligned}
$$

Similarly, substituting (A15) into (A17) and rearranging terms yields

$$
\begin{aligned}
& {\left[w_{1}\left(b_{0}\right)-b_{1}\right] K_{1}\left(b_{0}\right)\left[G_{\beta}\left(b_{1}\right)-G_{\beta}\left(b_{0}\right)\right]} \\
& \quad+\eta \int_{\left(-\infty, b_{0}\right]}\left[\left[w_{\Delta}(b)-b_{0}\right]\left[K_{1}(b)-K_{0}(b)\right]-\left[b_{1}-b_{0}\right] K_{1}(b)\right] P_{\beta}(d b) \geqslant 0 .
\end{aligned}
$$

We will show that relations (A18) and (A19) and the restrictions of Lemma 4 are inconsistent with relation (A14).

Since Lemma 4 (a) implies $w_{\Delta}(b) \leqslant w_{\Delta}\left(b_{0}\right), b \leqslant b_{0}$ a.s. $-P_{\beta}$, relation (A19) implies

$$
\begin{aligned}
& {\left[w_{1}\left(b_{0}\right)-b_{1}\right] K_{1}\left(b_{0}\right)\left[G_{\beta}\left(b_{1}\right)-G_{\beta}\left(b_{0}\right)\right]} \\
& \quad+\eta \int_{\left(-\infty, b_{0}\right]}\left[\left[w_{\Delta}\left(b_{0}\right)-b_{0}\right]\left[K_{1}(b)-K_{0}(b)\right]-\left[b_{1}-b_{0}\right] K_{1}(b)\right] P_{\beta}(d b) \geqslant 0 .
\end{aligned}
$$

Also, since Lemma 4(a) and relation (A14) imply $w_{\Delta}\left(b_{0}\right)>b_{0}$, and Lemma 4(b) implies [ $K_{1}(b)-$ $\left.K_{0}(b)\right] K_{1}\left(b_{0}\right) \leqslant\left[K_{1}\left(b_{0}\right)-K_{0}\left(b_{0}\right)\right] K_{1}(b), b \leqslant b_{0}$ a.s. $P_{\beta}$, relation (A20) implies

$$
\begin{aligned}
& {\left[w_{1}\left(b_{0}\right)-b_{1}\right] K_{1}\left(b_{0}\right)\left[G_{\beta}\left(b_{1}\right)-G_{\beta}\left(b_{0}\right)\right] K_{1}\left(b_{0}\right)} \\
& +\eta\left[\left[w_{\Delta}\left(b_{0}\right)-b_{0}\right]\left[K_{1}\left(b_{0}\right)-K_{0}\left(b_{0}\right)\right]\right. \\
& \left.\quad-\left[b_{1}-b_{0}\right] K_{1}\left(b_{0}\right)\right] \int_{\left(-\infty, b_{0}\right]} K_{1}(b) P_{\beta}(d b) \geqslant 0 .
\end{aligned}
$$

Finally, since Lemma $4(\mathrm{~b})$ implies $K_{1}(b) \geqslant K_{1}\left(b_{0}\right), b \leqslant b_{0}$ a.s. $P_{\beta}$, relation (A15) implies $\left[w_{1}\left(b_{0}\right)\right.$ $\left.b_{1}\right] K_{1}\left(b_{0}\right)>0$, it follows from (A21) that

$$
\begin{array}{r}
{\left[w_{1}\left(b_{0}\right)-b_{1}\right] K_{1}\left(b_{0}\right)\left[G_{\beta}\left(b_{1}\right)-G_{\beta}\left(b_{0}\right)\right]+\eta\left[\left[w_{\Delta}\left(b_{0}\right)-b_{0}\right]\left[K_{1}\left(b_{0}\right)-K_{0}\left(b_{0}\right)\right]\right.} \\
\left.-\left[b_{1}-b_{0}\right] K_{1}\left(b_{0}\right)\right] G_{\beta}\left(b_{0}\right) \geqslant 0 .
\end{array}
$$

Multiplying (A19) by $G_{\beta}\left(b_{0}\right)$, (A21) by $G_{\alpha}\left(b_{0}\right) / \eta$, and subtracting then implies $\left[G_{\beta}\left(b_{1}\right)-\right.$ $\left.G_{\beta}\left(b_{0}\right)\right] G_{\alpha}\left(b_{0}\right) \geqslant \eta\left[G_{\alpha}\left(b_{1}\right)-G_{\alpha}\left(b_{0}\right)\right] G_{\beta}\left(b_{0}\right)$, contradicting relation (A14). This proves part (a).

(b) If $R$ is independent of $(V, X)$, then $K\left(b \mid b^{\prime}\right)=K(b)$. Let $v(b)=E[V \mid \beta=b]$ and $H(b)=$ $G_{\alpha}(b) K(b)$. Suppose $G_{\alpha}$ is strictly increasing on $\left(b^{\prime}, b^{\prime \prime}\right)$ with $b^{\prime}>0$. Then part (a) implies that $G_{\beta}$ is also strictly increasing on $\left(b^{\prime}, b^{\prime \prime}\right)$ so that Assumption 4 guarantees that $v(b)>b, b \in\left(b^{\prime}, b^{\prime \prime}\right)$ a.s. $-P_{\beta}$.

We establish first that $G_{\beta}$ and $H$ are continuous on $\left(b^{\prime}, b^{\prime \prime}\right)$. For $b, b+\varepsilon \in\left(b^{\prime}, b^{\prime \prime}\right)$, the zero profit condition (Lemma 1(b)) implies $(b+\varepsilon) G_{\beta}(b+\varepsilon)-b G_{\beta}(b)=\int_{(b, b+\varepsilon]} v(t) P_{\beta}(d t)$. Letting $\varepsilon \downarrow 0$ then yields $P_{\beta}(\{b\})=0$. Similarly the best response property for $\beta$ (Lemma $1(a)$ ) implies $[v(b-\varepsilon)-$ $b] H(b) \leqslant[v(b-\varepsilon)-b+\varepsilon] H(b-\varepsilon)$ or, equivalently, $[v(b-\varepsilon)-b+\varepsilon][H(b)-H(b \varepsilon)] \leqslant \varepsilon H(b)$. Letting $\varepsilon \downarrow 0$ then yields $H(b-\varepsilon) \uparrow H(b)$. 
Given the continuity of $G_{\beta}$, we may express the zero profit condition for $\alpha$ in terms of the Stieltjes integral, $b G_{\beta}(b)=b_{0} G_{\beta}\left(b_{0}\right)+\int_{b_{0}}^{b} v(t) d G_{\beta}(t), b \in\left[b^{\prime}, b^{\prime \prime}\right)$. Let $\pi(b)=v(b)-b$. Then, applying the formula for integration by parts yields

$$
\int_{b^{\prime}}^{b^{\prime \prime}} \pi(b) d G_{\beta}(b)=\int_{b^{\prime}}^{b^{\prime \prime}} G_{\beta}(b) d b .
$$

Now consider an arbitrary increasing sequence $b^{\prime}=b_{0}<b_{1}<\cdots<b_{n-1}<b_{n}=b^{\prime \prime}$. The best response condition for $\beta$ implies, for $i=1, \ldots, n$,

$$
\pi\left(b_{l}\right)\left[H\left(b_{i}\right)-H\left(b_{i-1}\right)\right] \geqslant\left[b_{i}-b_{i-1}\right] H\left(b_{i-1}\right)
$$

and

$$
\pi\left(b_{i-1}\right)\left[H\left(b_{i}\right)-H\left(b_{i-1}\right)\right] \leqslant\left[b_{i}-b_{i-1}\right] H\left(b_{i}\right) .
$$

Since $\pi$ is of bounded variation (the difference between two monotone functions) and $H$ is continuous, (A24) and (A25) imply

$$
\int_{b^{\prime}}^{b^{\prime \prime}} \pi(t) d H(t)=\int_{b^{\prime}}^{b^{\prime \prime}} H(t) d t, \quad b \in\left[b^{\prime}, b^{\prime \prime}\right] .
$$

Furthermore, since $G_{\beta}$ and $H$ are continuous and positive on $\left[b^{\prime}, b^{\prime \prime}\right]$, it follows from (A23) and (A26) that, for $b \in\left[b^{\prime}, b^{\prime \prime}\right]$,

$$
\int_{b^{\prime}}^{b^{\prime \prime}} \pi(t) d \log \left(G_{\beta}(t)\right)=b-b^{\prime},
$$

and

$$
\int_{b^{\prime}}^{b^{\prime \prime}} \pi(t) d \log (H(t))=b-b^{\prime} .
$$

Subtracting (A28) from (A27) and recalling that $\pi(b)>0, b \in\left[b^{\prime}, b^{\prime \prime}\right]$, it follows that $\int_{b}^{b \prime} d \log (H(t))$ $=\int_{b}^{b \prime} d \log \left(G_{\beta}(t)\right), b \in\left[b^{\prime}, b^{\prime \prime}\right]$. Upon evaluating this expression and substituting for $H$, we obtain part (b).

Q.E.D.

Proof of Theorem 2: We will show that $G_{\beta}\left(b^{*}\right)=G_{\beta}(0)$ implies $G_{\alpha}\left(b^{\prime}\right)=G_{\alpha}(0)$, for some $b^{\prime}>b^{*}$. Fix $b_{1}>b^{*}$. Since Assumptions 4 and 5 imply that $G_{\beta}(0)>0$, it follows from Assumptions 3 and 4 that $K\left(b_{1} \mid b\right) G_{\alpha}\left(b_{1}\right)>0$ a.s. $P_{\beta}$. The best response property for $\beta$ implies $E\left[V-b_{1} \mid R \leqslant b_{1}\right.$, $\beta=b] K\left(b_{1} \mid b\right) G_{\alpha}\left(b_{1}\right) \leqslant 0$ for $b \leqslant b^{*}$ a.s. $P_{\beta}$. Therefore, it follows from Assumption 5 that $\int_{\left(-\infty, b^{*}\right]} E\left[V-b_{1} \mid R \leqslant b_{1}, \beta=b\right] K\left(b_{1} \mid b\right) P_{\beta}(d b)<0$. The right continuity of $G_{\beta}$ then implies a $b^{\prime}>b^{*}$ such that, for $b_{2} \leqslant b^{\prime}, \int_{\left(-\infty, b_{2}\right]} E\left[V-b_{1} \mid R \leqslant b_{1}, \beta=b\right] K\left(b_{1} \mid b\right) P_{\beta}(d b)<0$ and hence from Lemma 4(a) that $\int_{\left(-\infty, b_{2}\right]} E\left[V-b_{2} \mid R \leqslant b_{2}, \beta=b\right] K\left(b_{2} \mid b\right) P_{\beta}(d b)<0$. The result then follows from the zero profit condition for $\alpha$ (Lemma 1(b)).

Q.E.D.

\section{REFERENCES}

Ashenfelter, Orley (1989): "How Auctions Work for Wine and Art," Journal of Economic Perspectives, 3, 23-36.

Engelbrecht-Wiggans, R., Paul R. Milgrom, and Robert J. Weber (1983): "Competitive Bidding and Proprietary Information," Journal of Mathematical Economics, 11, 161-169.

Gaskins, Darius W., JR. (1976): In Hearings before the Subcommittee on Monopolies and Commercial Law of the House Committee of the Judiciary, Energy Industry Investigation, Part I: Joint Ventures. Washington: Government Printing House, 207-241.

Hendricks, Kenneth, and Robert H. Porter (1988): "An Empirical Study of an Auction with Asymmetric Information," American Economic Review, 78, 865-883.

Hendricks, Kenneth, Robert H. Porter, and Richard H. Spady (1989): "Random Reservation Prices and Bidding Behavior in O.C.S. Drainage Auctions," Journal of Law and Economics, 32, S83-S106. 
Hendricks, Kenneth, Robert H. Porter, and Guofu Tan (1993): “Optimal Selling Strategies for Oil and Gas Leases with an Informed Buyer," American Economic Review: Papers and Proceedings, 83, 234-239.

Hendricks, Kenneth, Robert H. Porter, and C. A. Wilson (1990): "Auctions For Oil and Gas Leases with an Informed Bidder and a Random Reservation Price," C.M.S.E.S. Discussion Paper No. 910, Northwestern University.

Laffont, Jean-Jacoues, Herve Ossard, and Quang Vuong (1993): "Econometrics of First Price Auctions," mimeo, University of Toulouse.

McAfee, P., And Daniel Vincent (1992): "Updating the Reserve Price in Common-Value Auctions," American Economic Review: Papers and Proceedings, 82, 512-518.

Milgrom, Paul R., and Robert J. Weber (1982): "A Theory of Auctions and Competitive Bidding," Econometrica, 50, 1089-1122.

- (1985): "Distributional Strategies for Games with Incomplete Information," Mathematics of Operations Research, 10, 619-632.

WeVERBERGH, M. (1979): "Competitive Bidding with Asymmetric Information Reanalyzed," Management Science, 25, 291-294.

WiLson, RoBert J. (1967): "Competitive Bidding with Asymmetric Information," Management Science, 13, 816-820. 
http://www.jstor.org

\title{
LINKED CITATIONS
}

- Page 1 of 2 -

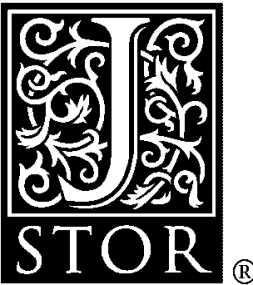

You have printed the following article:

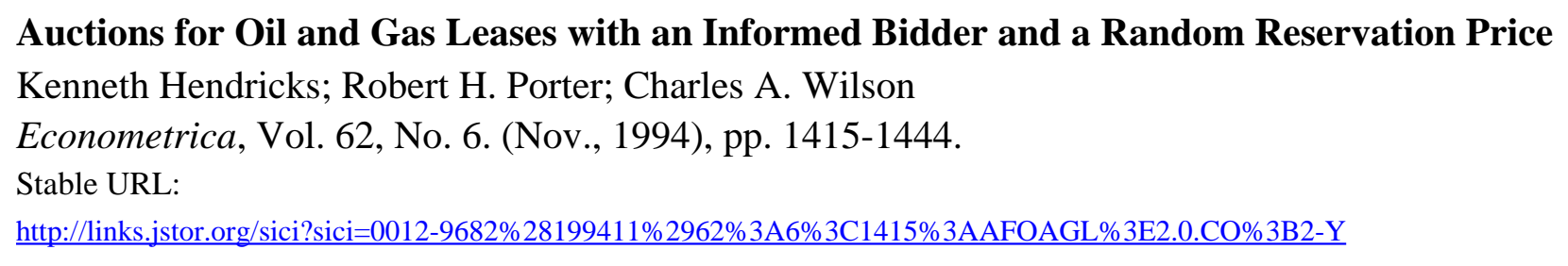

This article references the following linked citations. If you are trying to access articles from an off-campus location, you may be required to first logon via your library web site to access JSTOR. Please visit your library's website or contact a librarian to learn about options for remote access to JSTOR.

\section{References}

\author{
How Auctions Work for Wine and Art \\ Orley Ashenfelter \\ The Journal of Economic Perspectives, Vol. 3, No. 3. (Summer, 1989), pp. 23-36. \\ Stable URL: \\ http://links.jstor.org/sici?sici=0895-3309\%28198922\%293\%3A3\%3C23\%3AHAWFWA\%3E2.0.CO\%3B2-5
}

\author{
An Empirical Study of an Auction with Asymmetric Information \\ Kenneth Hendricks; Robert H. Porter \\ The American Economic Review, Vol. 78, No. 5. (Dec., 1988), pp. 865-883. \\ Stable URL: \\ http://links.jstor.org/sici?sici=0002-8282\%28198812\%2978\%3A5\%3C865\%3AAESOAA\%3E2.0.CO\%3B2-H \\ Random Reservation Prices and Bidding Behavior in OCS Drainage Auctions \\ Kenneth Hendricks; Robert H. Porter; Richard H. Spady \\ Journal of Law and Economics, Vol. 32, No. 2, Part 2, Empirical Approaches to Market Power: A \\ Conference Sponsored by the University of Illinois and the Federal Trade Commission. (Oct., 1989), \\ pp. S83-S106. \\ Stable URL: \\ http://links.jstor.org/sici?sici=0022-2186\%28198910\%2932\%3A2\%3CS83\%3ARRPABB\%3E2.0.CO\%3B2-F
}


http://www.jstor.org

\section{LINKED CITATIONS \\ - Page 2 of 2 -}

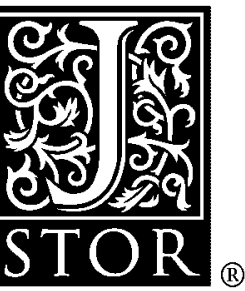

\section{Optimal Selling Strategies for Oil and Gas Leases with an Informed Buyer}

Kenneth Hendricks; Robert H. Porter; Guofu Tan

The American Economic Review, Vol. 83, No. 2, Papers and Proceedings of the Hundred and Fifth Annual Meeting of the American Economic Association. (May, 1993), pp. 234-239.

Stable URL:

http://links.jstor.org/sici?sici=0002-8282\%28199305\%2983\%3A2\%3C234\%3AOSSFOA\%3E2.0.CO\%3B2-1

\section{Updating the Reserve Price in Common-Value Auctions}

R. Preston McAfee; Daniel Vincent

The American Economic Review, Vol. 82, No. 2, Papers and Proceedings of the Hundred and Fourth Annual Meeting of the American Economic Association. (May, 1992), pp. 512-518.

Stable URL:

http://links.jstor.org/sici?sici=0002-8282\%28199205\%2982\%3A2\%3C512\%3AUTRPIC\%3E2.0.CO\%3B2-9

\section{A Theory of Auctions and Competitive Bidding}

Paul R. Milgrom; Robert J. Weber

Econometrica, Vol. 50, No. 5. (Sep., 1982), pp. 1089-1122.

Stable URL:

http://links.jstor.org/sici?sici=0012-9682\%28198209\%2950\%3A5\%3C1089\%3AATOAAC\%3E2.0.CO\%3B2-E

\section{Competitive Bidding with Asymmetric Information Reanalyzed}

M. Weverbergh

Management Science, Vol. 25, No. 3. (Mar., 1979), pp. 291-294.

Stable URL:

http://links.jstor.org/sici?sici=0025-1909\%28197903\%2925\%3A3\%3C291\%3ACBWAIR\%3E2.0.CO\%3B2-3

\section{Competitive Bidding with Asymmetric Information}

Robert B. Wilson

Management Science, Vol. 13, No. 11, Series A, Sciences. (Jul., 1967), pp. 816-820.

Stable URL:

http://links.jstor.org/sici?sici=0025-1909\%28196707\%2913\%3A11\%3C816\%3ACBWAI\%3E2.0.CO\%3B2-L 